\title{
A Color Vision-Based Lane Tracking System for Autonomous Driving on Unmarked Roads
}

\author{
MIGUEL ANGEL SOTELO AND FRANCISCO JAVIER RODRIGUEZ \\ Department of Electronics, University of Alcala, Alcalá de Henares, Madrid, Spain \\ michael@depeca.uah.es \\ fjrs@depeca.uah.es \\ LUIS MAGDALENA \\ Department of Applied Mathematics, Technical University, Madrid, Spain \\ 1layos@mat.upm.es \\ LUIS MIGUEL BERGASA AND LUCIANO BOQUETE \\ Department of Electronics, University of Alcala, Alcalá de Henares, Madrid, Spain \\ bergasa@depeca.uah.es \\ luciano@depeca.uah.es
}

\begin{abstract}
This work describes a color Vision-based System intended to perform stable autonomous driving on unmarked roads. Accordingly, this implies the development of an accurate road surface detection system that ensures vehicle stability. Although this topic has already been documented in the technical literature by different research groups, the vast majority of the already existing Intelligent Transportation Systems are devoted to assisted driving of vehicles on marked extra urban roads and highways. The complete system was tested on the BABIECA prototype vehicle, which was autonomously driven for hundred of kilometers accomplishing different navigation missions on a private circuit that emulates an urban quarter. During the tests, the navigation system demonstrated its robustness with regard to shadows, road texture, and weather and changing illumination conditions.
\end{abstract}

Keywords: color vision-based lane tracker, unmarked roads, unsupervised segmentation

\section{Introduction}

The main issue addressed in this work deals with the design of a vision-based algorithm for autonomous vehicle driving on unmarked roads.

\subsection{Motivation for Autonomous Driving Systems}

The deployment of Autonomous Driving Systems is a challenging topic that has focused the interest of research institutions all across the world since the mid eighties. Apart from the obvious advantages related to safety increase, such as accident rate reduction and human life savings, there are other benefits that could clearly derive from automatic driving. Thus, on one hand, vehicles keeping a short but reliable safety distance by automatic means allow to increase the capacity of roads and highways. This inexorably leads to an optimal use of infrastructures. On the other hand, a remarkable saving in fuel expenses can be achieved by automatically controlling vehicles velocity so as to keep a soft acceleration profile. Likewise, automatic cooperative driving of vehicle fleets involved in the 
transportation of heavy loads can lead to notable industrial cost reductions.

\subsection{Autonomous Driving on Highways and Extraurban Roads}

Although the basic goal of this work is concerned with the development of an Autonomous Driving System for unmarked roads, the techniques deployed for lane tracking in this kind of scenarios are similar to those developed for road tracking in highways and structured roads, as long as they face common problems. Nonetheless, most of the research groups currently working on this topic focus their endeavors on autonomously navigating vehicles on structured roads, i.e., marked roads. This allows to reduce the navigation problem to the localization of lane markers painted on the road surface. That's the case of some well known and prestigious systems such as RALPH (Pomerleau and Jockem, 1996) (Rapid Adapting Lateral Position Handler), developed on the Navlab vehicle at the Robotics Institute of the Carnegie Mellon University, the impressive unmanned vehicles developed during the last decade by the research groups at the UBM (Dickmanns et al., 1994; Lutzeler and Dickmanns, 1998) and Daimler-Benz (Franke et al., 1998), or the GOLD system (Bertozzi and Broggi, 1998; Broggi et al., 1999) implemented on the ARGO autonomous vehicle at the Universita di Parma. All these systems have widely proved their validity on extensive tests carried out along thousand of kilometers of autonomous driving on structured highways and extraurban roads. The effectivity of these results on structured roads has led to the commercialization of some of these systems as driving aid products that provide warning signals upon lane depart. Some research groups have also undertaken the problem of autonomous vision based navigation on completely unstructured roads. Among them are the SCARF and UNSCARF systems (Thorpe, 1990) designed to extract the road shape basing on the study of homogeneous regions from a color image. The ALVINN (Autonomous Land Vehicle In a Neural Net) (Pomerleau, 1993 ) system is also able to follow unmarked roads after a proper training phase on the particular roads where the vehicle must navigate. The group at the Universitat der Bundeswehr, Munich, headed by E. Dickmanns has also developed a remarkable number of works on this topic since the early 80's. Thus, autonomous guidance of vehicles on either marked or unmarked roads demonstrated its first results in Dickmanns and Zapp
(1986) and Dickmanns and Mysliwetz (1992) where nine road and vehicle parameters were recursively estimated following the 4D approach on 3D scenes. More recently, a combination of on- and off-road driving was achieved in Gregor et al. (2001) using the EMSvision (Expectation-based Multifocal Saccadic vision) system, showing its wide range of maneuvering capabilities as described in Gregor et al. (2001). Likewise, another similar system can be found in Lutzeler and Dickmanns (2000) and Gregor et al. (2002), where a real autonomous system for Intelligent Navigation in a network of unmarked roads and intersections is designed and implemented using edge detectors for lane tracking. The vehicle is equipped with a four camera vision system, and can be considered as the first completely autonomous vehicle capable to successfully perform some kind of global mission in an urban-like environment, also based on the EMS-vision system. On the other hand, the work developed by the Department of Electronics at the University of Alcala (UAH) in the field of Autonomous Vehicle Driving started in 1993 with the design of a vision based algorithm for outdoor environments (Rodriguez et al., 1998) that was implemented on an industrial fork lift truck autonomously operated on the campus of the UAH. After that, the development of a vision-based system (Sotelo et al., 2001; De Pedro et al., 2001) for Autonomous Vehicle Driving on unmarked roads was undertaken until reaching the results presented in this paper. The complete navigation system was implemented on BABIECA, an electric Citroen Berlingo commercial prototype as depicted in Fig. 1. The vehicle is equipped with a color camera, a DGPS receiver, two computers, and the necessary electronic equipment to allow for automatic actuation on the steering wheel, brake and acceleration pedals. Thus, complete lateral and longitudinal automatic actuation is issued during navigation. Real tests were carried out on a private circuit emulating an urban quarter, composed of streets and intersections (crossroads), located at the Instituto de Automática Industrial del CSIC in Madrid, Spain. Additionally, a live demonstration exhibiting the system capabilities on autonomous driving was also carried out during the IEEE Conference on Intelligent Vehicles 2002, in a private circuit located at Satory (Versailles), France.

The work described in this paper is organized in the following sections: Section 2 describes the color vision based algorithm for lane tracking. Section 3 provides some global results, and finally, concluding remarks are presented in Section 4. 


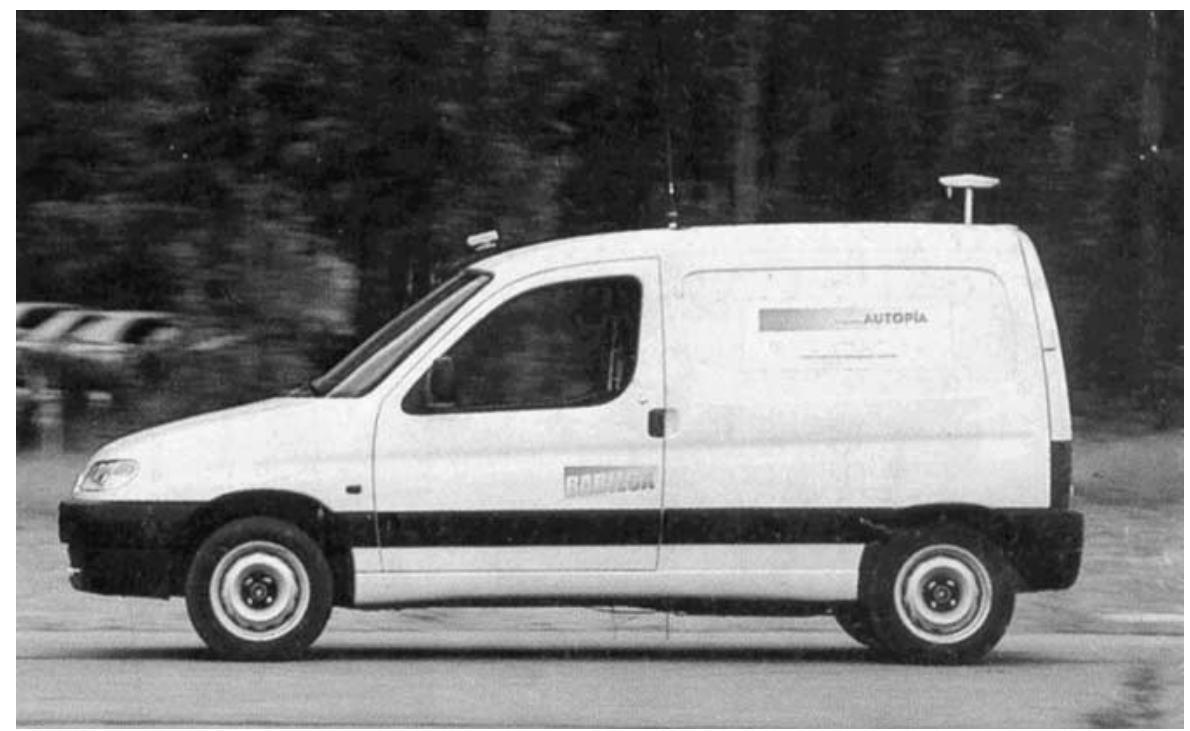

Figure 1. Babieca autonomous vehicle.

\section{Lane Tracking}

As described in the previous section, the main goal of this work is to robustly track the lane of any kind of road (structured or not). This includes the tracking of non structured roads, i.e., roads without lane markers painted on them.

\subsection{Region of Interest}

The original $480 \times 512$ incoming image acquired by a color camera is in real time re-scaled to a low resolution $60 \times 64$ image, by making use of the system hardware capabilities. It inevitably leads to a decrement in pixel resolution that must necessarily be assessed. Thus, the maximum resolution of direct measurements is between $4 \mathrm{~cm}$, at a distance of $10 \mathrm{~m}$, and $8 \mathrm{~cm}$ at $20 \mathrm{~m}$. Nonetheless, the use of temporal filtering techniques (as described in the following sections) allows to obtain finer resolution estimations. As discussed in Bertozzi et al. (2000) due to the existence of physical and continuity constraints derived from vehicle motion and road design, the analysis of the whole image can be replaced by the analysis of a specific portion of it, namely the region of interest. In this region, the probability of finding the most relevant road features is assured to be high by making use of a priori knowledge on the road shape, according to the parabolic road model proposed. Thus, in most cases the region of interest is reduced to some portion of image surrounding the road edges estimated in the previous iteration of the algorithm. This is a valid assumption for road tracking applications heavily relying on the detection of lane markers that represent the road edges. This is not the case of the work presented in this paper, as the main goal is to autonomously navigate on completely unstructured roads (including rural paths, etc). As will be later described, color and shape features are the key characteristics used to distinguish the road from the rest of elements in the image. This leads to a slightly different concept of region of interest where the complete road must be entirely contained in the region under analysis.

On the other hand, the use of a narrow focus of attention surrounding the previous road model is strongly discarded due to the unstable behavior exhibited by the segmentation process in practice (more detailed justification will be given in the next sections). A rectangular region of interest of $36 \times 64$ pixels covering the nearest $20 \mathrm{~m}$ ahead of the vehicle is proposed instead, as shown in Fig. 2. This restriction permits to remove nonrelevant elements from the image such as the sky, trees, buildings, etc.

\subsection{Road Features}

The combined use of color and shape restrictions provides the essential information required to drive on non structured roads. Prior to the segmentation of the 


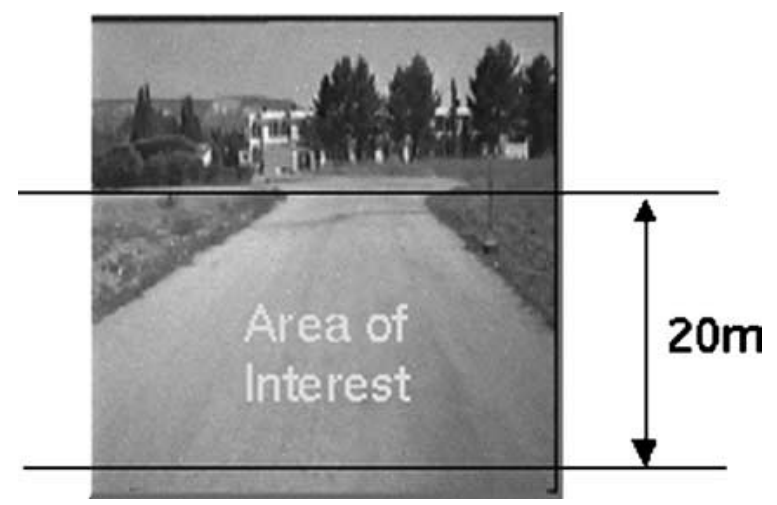

Figure 2. Area of interest.

image, a proper selection of the most suitable color space becomes an outstanding part of the process. On one hand, the RGB color space has been extensively tested and used in previous road tracking applications on non-structured roads (Thorpe, 1990; Crisman and Thorpe, 1991; Rodriguez et al., 1998). Nevertheless, the use of the RGB color space has some well known disadvantages, as mentioned next. It is non-intuitive and non-uniform in color separation. This means that two relatively close colors can be very separated in the RGB color space. RGB components are slightly correlated. A color can not be imagined from its RGB components. On the other hand, in some applications the RGB color information is transformed into a different color space where the luminance and chrominance components of the color are clearly separated from each other. This kind of representation benefits from the fact that the color description model is quite oriented to human perception of colors. Additionally, in outdoor environments the change in luminance is very large due to the unpredictable and uncontrollable weather conditions, while the change in color or chrominance is not that relevant. This makes highly recommendable the use of a color space where a clear separation between intensity (luminance) and color (chrominance) information can be established.

The HSI (Hue, Saturation and Intensity) color space constitutes a good example of this kind of representation, as it permits to describe colors in terms that can be intuitively understood. A human can easily recognize basic color attributes: intensity (luminance or brightness), hue or color, and saturation (Ikonomakis et al., 2000). Hue represents the impression related to the predominant wavelength in the perceived color stimulus. Saturation corresponds to the color relative purity, and thus, non saturated colors are gray scale colors. Intensity is the amount of light in a color. The maximum intensity is perceived as pure white, while the minimum intensity is pure black. Some of the most relevant advantages related to the use of the HSI color space are discussed below. It is closely related to human perception of colors, having a high power to discriminate colors, specially the hue component. The difference between colors can be directly quantified by using a distance measure. Transformation from the RGB color space to the HSI color space can be made by means of Eqs. (1) and (2), where V1 and V2 are intermediate variables containing the chrominance information of the color.

$$
\begin{gathered}
{\left[\begin{array}{c}
I \\
V_{1} \\
V_{2}
\end{array}\right]=\left[\begin{array}{ccc}
\frac{1}{3} & \frac{1}{3} & \frac{1}{3} \\
\frac{-1}{\sqrt{6}} & \frac{-1}{\sqrt{6}} & \frac{2}{\sqrt{6}} \\
\frac{1}{\sqrt{6}} & \frac{-2}{\sqrt{6}} & \frac{1}{\sqrt{6}}
\end{array}\right] \cdot\left[\begin{array}{c}
R \\
G \\
B
\end{array}\right]} \\
H=\arctan \frac{V_{2}}{V_{1}} \quad S=\sqrt{V_{1}^{2}+V_{2}^{2}}
\end{gathered}
$$

This transformation describes a geometrical approximation to map the RGB color cube into the HSI color space, as depicted in Fig. 4. As can be clearly appreciated from observation of Fig. 3, colors are distributed in a cylindrical manner in the HSI color space. A similar way to proceed is currently under consideration by performing a change in the coordinate frames so as to align with the $I$ axis, and compute one component along the $I$ axis and the other in the plane normal to the $I$ axis. This could save some computing time by avoiding going through the trigonometry.

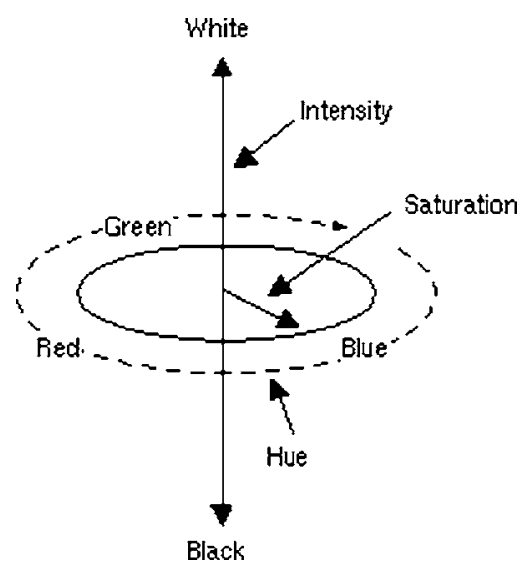

Figure 3. Mapping from the RGB cube to the HSI color space. 
Although the RGB color space has been successfully used in previous works dealing with road segmentation (Thorpe, 1990; Rodriguez et al., 1998), the HSI color space has exhibited superior performance in image segmentation problems as demonstrated in Ikonomakis et al. (2000). According to this, we propose the use of color features in the HSI color space as the basis to perform the segmentation of non-structured roads. A more detailed discussion supporting the use of the HSI color space for image segmentation in outdoor applications is extensively reported in Sotelo (2001).

\subsection{Road Model}

The use of a road model eases the reconstruction of the road geometry and permits to filter the data computed during the features searching process. Among the different possibilities found in the literature, models relaying on clothoids (Dickmanns et al., 1994) and polynomial expressions have extensively exhibited high performance in the field of road tracking. More concretely, the use of parabolic functions to model the projection of the road edges onto the image plane has been proposed and successfully tested in previous works (Schneiderman and Nashman, 1994). Parabolic models do not allow inflection points (curvature changing sign). This could lead to some problems in very snaky appearance roads. Nonetheless, the use of parabolic models has proved to suffice in practice for autonomous driving on two different test tracks including bended roads by using an appropriate lookahead distance as described in Sotelo (2003). On the other hand, some of the advantages derived from the use of a second order polynomial model are described below.

- Simplicity: a second order polynomial model has only three adjustable coefficients.

- Physical plausibility: in practice, any real stretch of road can be reasonably approximated by a parabolic function in the image plane. Discontinuities in the road model are only encountered in road intersections and, particularly, in crossroads.

According to this, we've adopted the use of second order polynomial functions for both the edges and the center of the road (the skeleton lines will serve as a reference trajectory from which the steering angle command will be obtained), as depicted in Fig. 5.
The adjustable parameters of the several parabolic functions are continuously updated at each iteration of the algorithm using a well known least squares estimator, as will be described later. Likewise, the road width is estimated basing on the estimated road model under the slowly varying width and flat terrain assumptions. The joint use of a polynomial road model and the previously mentioned constraints allows for simple mapping between the 2D image plane and the 3D real scene using one single camera.

\subsection{Road Segmentation}

Image segmentation must be carried out by exploiting the cylindrical distribution of color features in the HSI color space, bearing in mind that the separation between road and no road color characteristics is nonlinear. To better understand the most appropriate distance measure that should be used in the road segmentation problem consider again the decomposition of a color vector into its three components in the HSI color space, as illustrated in Fig. 4. According to the previous decomposition, the comparison between a pattern pixel denoted by $P_{p}$ and any given pixel $P_{i}$ can be directly measured in terms of intensity and chrominance distance, as depicted in Fig. 5.

From the analytical point of view, the difference between two color vectors in the HSI space can be

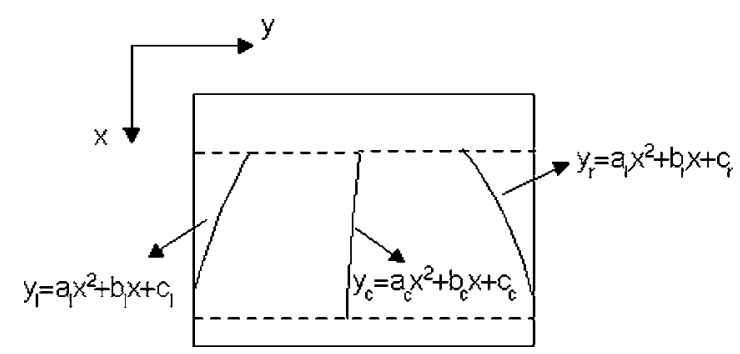

Figure 4. Road model.

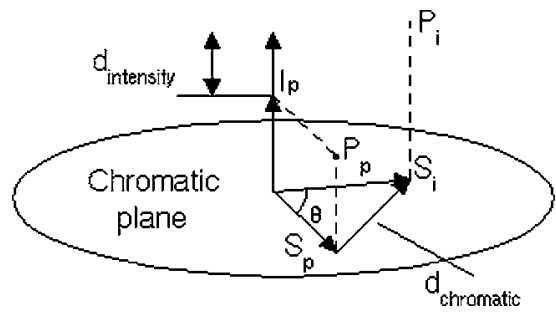

Figure 5. Color comparison in HSI space. 
established by computing the distances both in the chromatic plane, $d_{\text {chromatic }}$, and in the luminance axis, $d_{\text {intensity }}$, as described in Eqs. (3) and (4).

$$
\begin{aligned}
d_{\text {intensity }} & =\left|I_{p}-I_{i}\right| \\
d_{\text {chromatic }} & =\sqrt{\left(S_{p}\right)^{2}+\left(S_{i}\right)^{2}-2 S_{p} S_{i} \cos \theta}
\end{aligned}
$$

with

$$
\theta= \begin{cases}\left|H_{p}-H_{i}\right| & \text { if }\left|H_{p}-H_{i}\right|<180^{\circ} \\ 360^{\circ}-\left|H_{p}-H_{i}\right| & \text { if }\left|H_{p}-H_{i}\right|>180^{\circ}\end{cases}
$$

where $H_{p}, H_{i}, S_{p}, S_{i}, I_{p}$, and $I_{i}$ represent the Hue, Saturation and Intensity values of the pattern (p) and given (i) pixels, respectively. As can be readily derived from the previous equations, $d_{\text {chromatic }}$ measures the distance between two 2D color vectors in the chromatic plane while $d_{\text {intensity }}$ provides the luminance difference between the pattern pixel and the pixel under consideration. The cylindrical distribution of characteristics in the HSI color space must be suitably exploited in order to provide an appropriate segmentation method.

According to this, a cylindrical surface of separation between the road and non-road classes is proposed in an attempt to decouple chromatic changes from luminance changes, as the latter are much greater in outdoor environments despite intensity is not a determinant characteristic in the color segmentation process. In other words, any given pixel $i$ will be classified as road if the chromatic distance ( $\left.d_{\text {chromatic }}\right)$ to the color pattern is below some threshold $T_{\text {chrom }}$, and the intensity distance ( $\left.d_{\text {intensity }}\right)$ is lower than some $T_{\text {int }}$. This constraints the road pixels features in a cylinder around the pattern color vector. Despite hue is the most powerful color attribute for segmentation purposes, this feature is not significant when the intensity is extremely low or extremely high. On the other hand, hue is unstable when saturation is very low, as demonstrated in Gonzales and Wood (1992). According to this, pixels are divided into chromatic and achromatic as proposed in Ikonomakis et al. (2000). Any given pixel is considered to be achromatic if its intensity is below $10 \%$ or above $90 \%$ of the maximum normalized intensity, or if its saturation is under $10 \%$ of the maximum normalized saturation, as expressed in Eq. (6).

achromatic pixels:

$$
I>0.9 I_{\max } \text { or } I<0.1 I_{\max } \text { or } S<0.1 S_{\max }
$$

where $I_{\max }$ and $S_{\max }$ represent the maximum normalized intensity and saturation values, respectively. Achromatic pixels are segmented according to its intensity value alone. Obviously, non-achromatic pixels are automatically categorized as chromatic. The segmentation of chromatic pixels is accomplished by applying the previously proposed cylindrical separation in the HSI color space.

2.4.1. Adding Spatial Constraints. The quality of road segmentation can be strongly enhanced by adding spatial constraints according to the parabolic model used to describe the road edges. Consider the polynomial curve $y_{c}$ describing the trajectory of the central points of the road, projected on the image plane as depicted in Fig. 6. In an intuitive manner, the probability that a pixel is segmented as road is high if the pixel is located close to the previous road model (as estimated in the last iteration of the algorithm), described by $y_{c}(t-1)$. This is particularly true for short computing time algorithms considering that in practice, due to physical constraints both in road curvature design and in vehicle dynamics, either the road width or the temporal road model $y_{c}(t)$ vary gradually between two consecutive images. The last statement can be regarded as the slow varying road width assumption, widely used in previous works on road tracking (Kim et al., 1995; Pomerleau and Jockem, 1996).

Incorporating spatial constraints in the segmentation stage is not a trivial process that can be accomplished in several ways. For each pixel in the image the dimension of the cylindrical surface used for segmentation is modified according to the distance from the pixel under consideration to the previously estimated road model $y_{c}(t-1)$, and thus, threshold values $T_{\text {chrom }}$ and $T_{\text {int }}$ are modulated as a function of such distance. This turns the segmentation stage into a position dependant process.

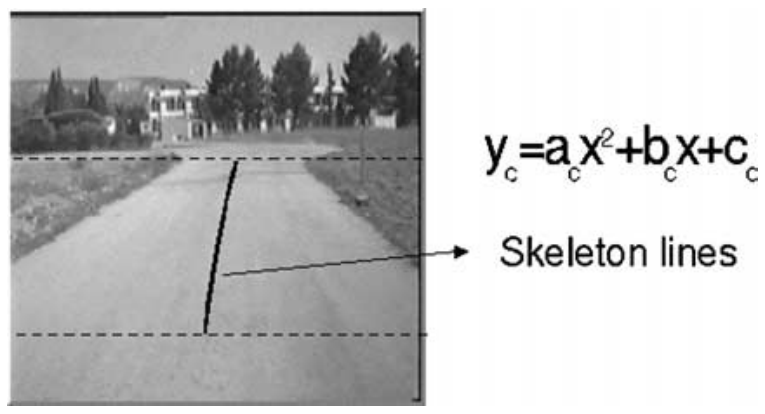

Figure 6. Parabolic model of the central points of the road. 


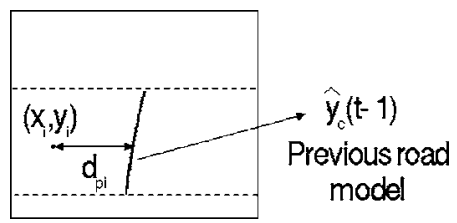

Figure 7. Computation of distance between pixel $i$ and the previously estimated road model.

The distance $d_{p i}$ between any given pixel $i$ with image coordinates $\left(x_{i}, y_{i}\right)$ and the previous road model $y_{c}(t-1)$ is computed on the image plane as described by Eq. (7). Graphically, the process is illustrated in Fig. 7.

$d_{p i}=\left|y_{i}-\left[a_{c}(t-1) \cdot x_{i}^{2}+b_{c}(t-1) \cdot x_{i}+c_{c}(t-1)\right]\right|$

The real distance $d_{i}$ between the point corresponding to the projection of pixel $i$ on the 3D scene, and the skeleton lines of the road is computed using the camera calibration parameters under the flat terrain assumption. This could be affected by the pitch angle of the vehicle, as small changes do have a noticeable effect, specially in minor roads with less smooth surface. In such cases, the planar assumption would lead to some errors in the road model computation. To compensate for this problem the use of a stabilization system is strongly recommended. Threshold values $T_{\text {chrom }}$ and $T_{\text {int }}$ are then modified for each pixel according to distance $d_{i}$ previously computed. The theoretical justification for this is the following. For each iteration of the segmentation algorithm, road pixels are expected to appear near (or around) the previously estimated skeleton lines of the road. Thus, a pixel located far away from the previous road model is most likely a pixel not contained in the current road, even if their color properties are similar. In other words, the probability of such pixel to belong to the current road is small. Accordingly, the proposed modification is accomplished so as to provide low threshold values for pixels far away from the previous road model. Thus, for pixels clearly located out of the road trajectory, the chromatic and luminance distances to the road pattern color features should be very small in order to effectively be segmented as part of the road. On the contrary, for pixels near the skeleton lines of the previous road model those distances are admitted to be larger. This means that the probability of a pixel to be segmented as road decreases as the distance between the pixel and the road model increases. Clearly, the goal of this technique is to reinforce the segmentation of road pixels near the skeleton lines of the road. From the analytical point of view, the proposal reflects an exponential variation of threshold values $T_{\text {chrom }}$ and $T_{\text {int }}$ for each individual pixel $i$ as a function of $d$, according to the expression in Eq. (8).

$$
\begin{aligned}
& \Psi_{c}(d)=\exp ^{\frac{-K \cdot d}{\hat{W}(t-1)}} \cdot T_{c}(t-1) \\
& \Psi_{I}(d)=\exp ^{\frac{-K \cdot d}{\hat{W}(t-1)}} \cdot T_{I}(t-1)
\end{aligned}
$$

where $\Psi_{c}(d)$ and $\Psi_{I}(d)$ represent the threshold values for the chromatic and luminance distances, respectively, for a pixel located at a distance $d$ from the previous model, $T_{c}(t-1)$ and $T_{I}(t-1)$ are the maximum threshold values estimated in the previous iteration, and $K$ is an empirically determined parameter devised to control the threshold value, particularly for pixels located in the surroundings of the road edges. In practice, $K$ is determined so that the threshold value is $70 \%$ of the maximum threshold, for pixels located at a distance from the model $d_{w}=\hat{W}(t-1) / 2$. This empirical value has demonstrated to be appropriate after practical trials and to allow for a stable segmentation process, yielding the numerical value for variable $K$ depicted in Eq. (9).

$$
K=-2 \cdot \ln (70 / 100)
$$

A chromatic pixel $i$ is classified as road if it simultaneously verifies that $d_{\text {chrom }}<\Psi_{c}(d)$ and $d_{\text {int }}<\Psi_{I}(d)$, while an achromatic pixel is segmented as road if the single condition $d_{\text {int }}<\Psi_{I}(d)$ is satisfied. Obviously, the initial choice of $T_{c}(0)$ and $T_{I}(0)$ becomes a critical decision whose justification is fully detailed in the next section. On the other hand, the maximum threshold values $T_{c}(t)$ and $T_{I}(t)$ must be dynamically updated so as to adapt the segmentation process to changing color and luminance conditions. To carry out this, the root mean squared values of the chromatic and luminance distances to the road pattern, $d_{\text {chrom,rms }}$ and $d_{\text {int,rms }}$, are computed for each pixel classified as road. The maximum threshold values for the next iteration, $T_{c}(t+1)$ and $T_{I}(t+1)$, are calculated as described in Eq. 10 depending on $d_{\text {chrom,rms }}(t), d_{\text {int,rms }}(t)$, and an exponential factor essential to guarantee the stability of the segmentation process. This leads to threshold values for the next iteration, $\Psi_{c}(d)_{\mid t+1}$ and $\Psi_{I}(d)_{\mid t+1}$, exactly equal to $d_{\text {chrom,rms }}(t)$ and $d_{\text {int,rms }}(t)$, respectively, for pixels 
located on the road edges $\left(d_{\mid t+1}=\hat{W}(t) / 2\right)$.

$$
\begin{aligned}
& T_{c}(t+1)=d_{\mathrm{chrom}, \mathrm{rms}}(t) \cdot e^{\frac{K}{2}} \\
& T_{I}(t+1)=d_{\mathrm{int}, \mathrm{rms}}(t) \cdot e^{\frac{K}{2}}
\end{aligned}
$$

2.4.2. Initial Segmentation. An initial road pattern color vector must be determined so as to provide correct features reference for the first iteration of the classification process. As can be easily imagined, no universal road features vector could be a priori considered regarding that the system should work in a long variety of different and complex scenarios, ranging from urban roads to rural paths. An initial road pattern color vector is computed from the information contained in the first image in a non-supervised manner, assuming that the road is well within the field of view (at least one edge of the road should be visible). The color features of pixels near the skeleton lines of the initial road model are averaged to obtain the road pattern, as the probability that those pixels belong to the road is quite high. This leads to the need for determining the initial equation of the skeleton lines of the road $y_{c}(0)=a_{c}(0) \cdot x^{2}+b_{c}(0) \cdot x+c_{c}(0)$. An iterative procedure is then started aiming at finding the initial road model. This model is intended to serve as the initial reference around which a set of pixels is selected to compute a candidate road pattern color vector. Seven a priori road models are utilized for this purpose, as depicted in Fig. 8. The choice of these models has been made according to the position of the camera and its calibration parameters.

For each model, a set of $M$ pixels is randomly selected in a surrounding of $1 \mathrm{~m}$ around the skeleton lines of the model. The HSI color features of the $M$ selected pixels are averaged to yield a candidate road pattern color vector for road model $i\left(I_{p i}, H_{p i}, S_{p i}\right)$, as indicated in Eq. (11), where a set of $M=10$ pixels has

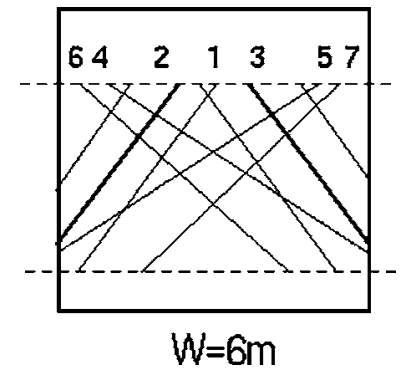

Figure 8. A priori road models used to determine the initial road pattern. experimentally proved sufficient to yield a representative road pattern color vector.

$$
\begin{aligned}
C_{i} & =\sum_{k=1}^{M} S_{k i} \cdot \cos \left(H_{k i}\right) \\
S_{i} & =\sum_{k=1}^{M} S_{k i} \cdot \sin \left(H_{k i}\right) \\
H_{p i} & =\arctan \left(S_{i} / C_{i}\right) \\
S_{p i} & =\sqrt{C_{i}^{2}+S_{i}^{2}} \\
I_{p i} & =\frac{1}{M} \sum_{k=1}^{M} I_{k i}
\end{aligned}
$$

The maximum threshold values $T_{c}(0)$ and $T_{I}(0)$ are determined according to the quadratic distances $d_{\text {chrom,rms }-p i}$ and $d_{\text {int,rms }-p i}$ between the $M$ selected pixels and the candidate road pattern vector for model $i$ $\left(H_{p i}, S_{p i}, I_{p i}\right)$, obtained as described in Eqs. (12) and (13).

$$
\begin{gathered}
d_{\text {chrom }, \mathrm{rms}-p i}=\sqrt{\frac{1}{M} \sum_{j=1}^{M} d_{\mathrm{jchrom}-p i}^{2}} \\
d_{\text {int,rms }-p i}=\sqrt{\frac{1}{M} \sum_{j=1}^{M} d_{\mathrm{jint}-p i}^{2}} \\
T_{c}(0)=d_{\text {chrom }, \mathrm{rms}-p i} \cdot e^{\frac{K}{2}} \\
T_{I}(0)=d_{\text {int }, \mathrm{rms}-p i} \cdot e^{\frac{K}{2}}
\end{gathered}
$$

where $d_{\mathrm{jchrom}-p i}$ and $d_{\mathrm{jint}-p i}$ represent respectively the chromatic and intensity distances between pixel $j$ and the candidate road pattern vector given by model $i$. The segmentation of the image is then carried out as described in the previous sections, using the candidate road pattern vector and an initial road width $W(0)=$ $6 m$ (which is the standard width for a two lanes urban road. Likewise, it is similar to the standard width for extraurban roads with only one lane for each driving direction, which are the only ones considered in this approach). The quality of the resulting segmentation is evaluated by means of $S_{0 i}$. Index $S_{0 i}$ is intended to validate the resulting segmentation by measuring the correlation between the initial road model $i$ and the road segmentation obtained using the candidate road pattern color vector computed from road model $i$. The process is graphically illustrated in Fig. 9.

Analytically, $S_{0 i}$ is defined as the number of road pixels in road model $i$ matching the road pixels in the 


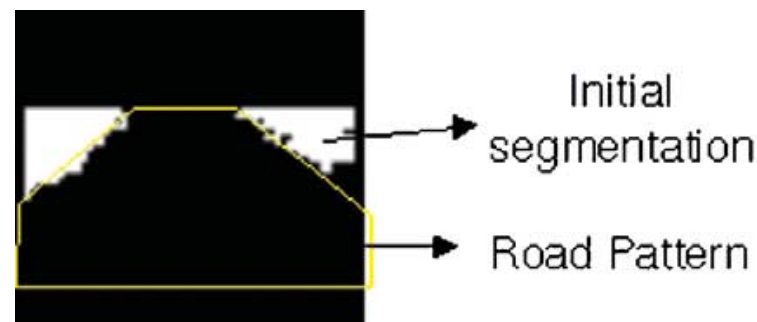

Figure 9. Comparison between road model $i$ and the initial road segmentation obtained using such model.

initial segmented image using such model. The final value is normalised by the total number of pixels in the image $(P)$, as Eq. (14) shows.

$$
S_{0 i}=\frac{1}{P} \sum_{k=1}^{P}\left[1-d_{H}\left(V_{k i}, V_{k 0 i}\right)\right]
$$

where $V_{k i}$ represents the binary value of segmented pixel $k$ ( 0 stands for road and 1 for no road), $V_{k 0 i}$ is the binary value of pixel $k$ in the initial segmentation using road model $i$, and $d_{H}$ is the Hamming distance. Thus, a value of $S_{0 i}$ close to unity represents a high correlation between the segmentation and model $i$, doubtless indicating that model $i$ is fit to represent the real shape of the road in the image plane. A minimum value $S_{0 i \text {, min }}=0.7$ is empirically established in order to validate the resulting segmentation. The validation process is recursively iterated until one of the $N$ a priori road models yields a proper segmentation result. If no a priori model succeeds in doing so, the width for all road models is modified in an amount of $\pm 5 \%$ the nominal width. If the model road width reaches a modification of $\pm 20 \%$ the nominal width without producing a valid segmentation, the iterative process is restarted from the standard value $W=6 \mathrm{~m}$ just where it started, until the appearance of the road allows for an appropriate initial segmentation. The complete algorithm to perform the initial segmentation is depicted in Fig. 10.

Experimental results have successfully proved the ability of the proposed strategy to carry out the initial segmentation, yielding reasonably neat binary images for arbitrary initial vehicle orientations, while keeping the processing time under $1 \mathrm{~ms}$ for most of the experiments conducted on a real scenario, as depicted in Fig. 11. To enhance the quality of the segmentation process, the resulting binary image is reinforced by a morphological opening operation (using a cross-shaped $3 \times 3$ structuring element) followed by the removal of small white blobs corresponding to segmentation

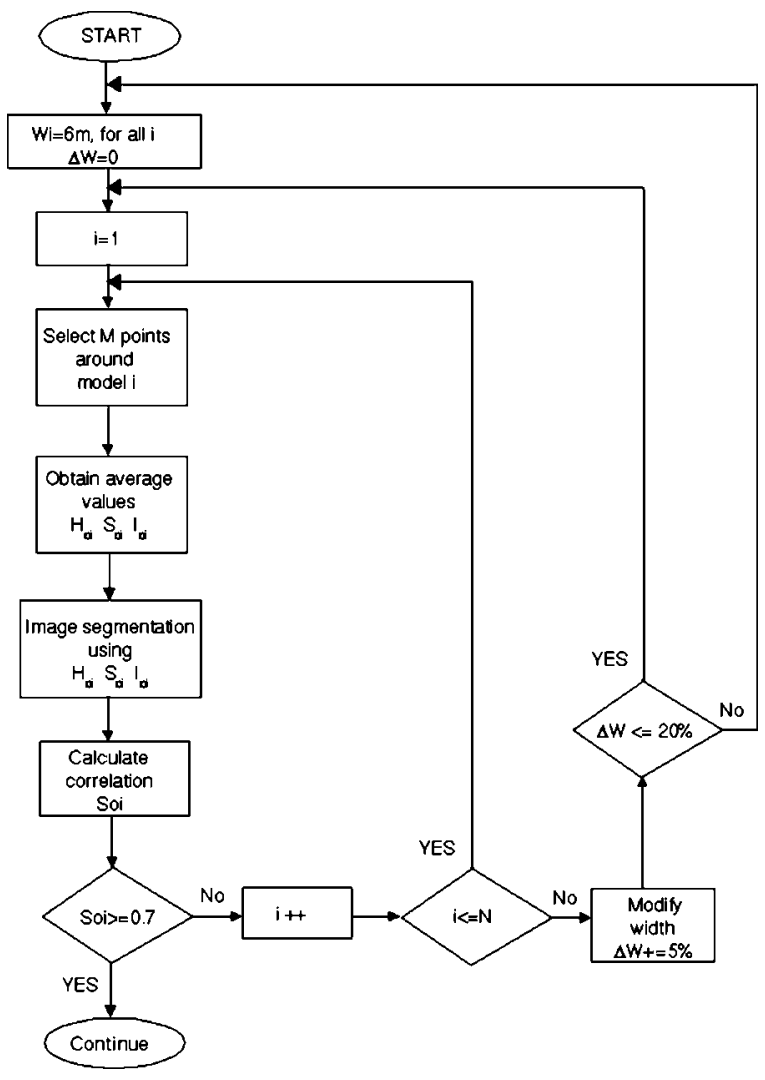

Figure 10. Algorithm for initial segmentation.

noise. The benefits derived from these operations can be graphically appreciated in Fig. 12.

\subsection{Handling Shadows and Brightness}

Shadows and brightness on the road are admittedly the greatest difficulty in vision based systems operating in outdoor environments (Bertozzi and Broggi, 1998). The problem affects the detection of both lane markings and road edges in general, becoming specially dangerous at some particular hours of the day when the sun directly shines onto the image plane, deriving in situations where loss of tracking occurs. In order to deal with this problem, some authors propose to improve the dynamic range of visual cameras (Bertozzi et al., 2000) so as to tackle strong luminance changes, when entering or exiting tunnels for instance, or to enhance the sensitiveness of cameras to the blue component of colors. A different approach undertakes alone the problem of shadows by attenuating their effects using an appropriate software pre-processing technique, 

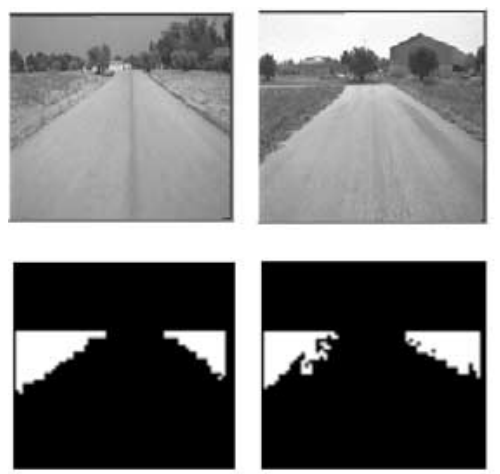

$t=156$ us
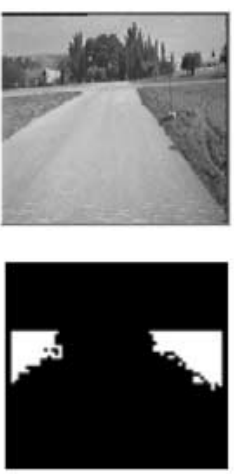

$t=141 \mu s$
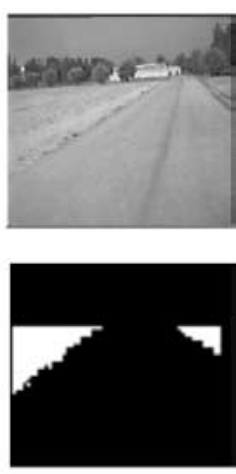

$t=146 u s$

Figure 11. Examples of initial segmentations.
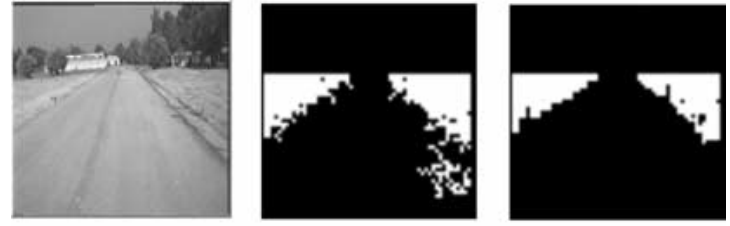

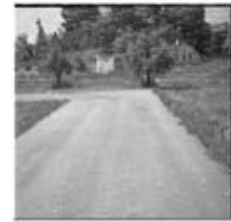

a)

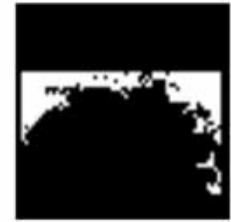

b)

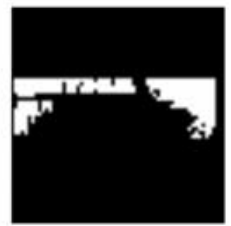

c)
Figure 12. Segmentation results after morphological postprocessing. (a) Original image. (b) Original segmentation. (c) Segmentation after morphological post-processing.

relaying on physical properties of shaded road pixels. On one hand, shaded road pixels exhibit lower intensity values than their neighbors corresponding to nonshaded road pixels. On the other hand, the normalized blue component is generally predominant over the normalized red and green components, as discussed in Pomerleau (1993). According to this, the addition of both the blue and the normalized blue components is then exploited to attenuate the effect of shadows, to some extent. This approach is intended to yield a low resolution gray scale image that serves as input for a neural network that directly obtains the vehicle turning angle, assuming that no color information is used thereinafter.

In the current work, a slightly different strategy is formulated so as to enhance the resulting segmentation against the effects of both shadows and brightness. This is realized by accounting for color properties of pixels located within the road edges, as estimated in the previous iteration of the algorithm, in an attempt to incorporate spatial constraints in the shadows and brightness attenuation process. Thus, color features of pixels located within the limits of the road, but classified as non-road after the segmentation process, are considered for brightness and shadows attenuation. Shaded pixels should exhibit an intensity value lower than the average intensity of road pixels, while presenting a predominant normalized blue component. Those pixels complying with the conditions previously described, and analytically expressed in Eq. (15), are assumed to belong to a shadow on the pavement and will be consequently reclassified as road pixels. It must be remarked that this is approximately true for any kind of real road, no matter the color of it.

$$
\begin{aligned}
b & \geq \frac{1}{3} \\
I & \leq I_{\text {road,avg }}-2 \cdot \sigma_{\text {road }}
\end{aligned}
$$

where $b$ stands for the normalized blue component; $I_{\text {road,avg }}$ represents the average intensity value of all road pixels, and $\sigma_{\text {road }}$ is the standard deviation of the intensity distribution of road pixels. This technique permits to enhance the road segmentation in presence of shadows, and remarkably contributes to improve the robustness of the color adaptation process, particularly in stretches of road largely covered by shadows. To graphically illustrate the benefits derived from this operation, Fig. 13 shows an example of road segmentation in presence of shadows. As can be appreciated, the road edges are neatly distinguished after the attenuation of shadows. 

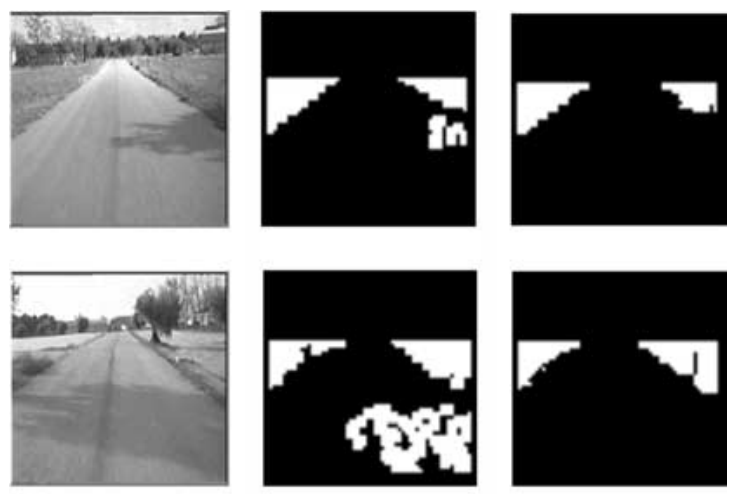

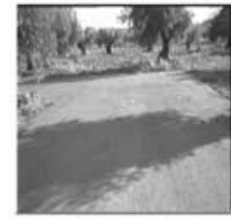

a)

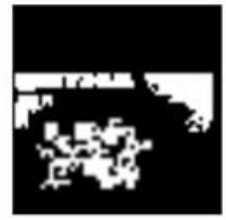

b)

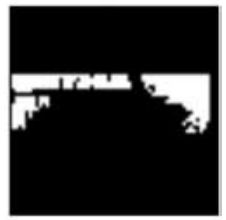

c)
Figure 13. Attenuation of shadows. (a) Original shaded images. (b) Segmentation without attenuation of shadows. (c) Segmentation after attenuation of shadows.

Analogously, a brightness attenuation technique has been devised. In this case, pixels initially classified as non-road but located within the road edges and exhibiting higher intensity values than the average road pixels, are assumed to correspond to brightness on the pavement caused by the sun, and consequently will be re-classified as road-pixels. Analytically the condition is formulated in Eq. (16).

$$
I \geq I_{\text {road, avg }}+2 \cdot \sigma_{\text {road }}
$$

where $I_{\text {road,avg }}$ and $\sigma_{\text {road }}$ are the same variables considered in Eq. (15). After applying the condition established by Eq. (16), white blobs due to brightness are removed from the segmentation as depicted in Fig. 14.

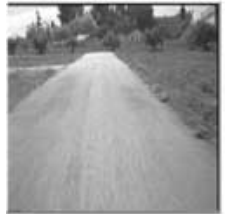

a)

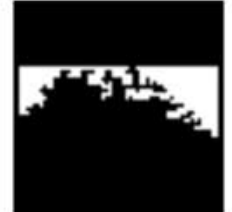

b)

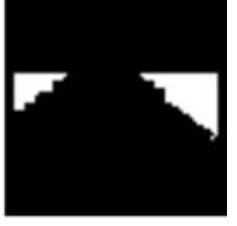

c)
Figure 14. Brightness attenuation. (a) Original image with brightness on the pavement. (b) Segmentation without brightness attenuation. (c) Segmentation after brightness attenuation.
The improvement achieved by attenuating both brightness and shadows, as described, permits to handle real images in real and complex situations with an extraordinary high performance, becoming an outstanding point of this work.

\subsection{Estimation of Road Edges and Width}

The estimation of the skeleton lines of the road and its edges is carried out basing on parabolic functions, as previously described. These polynomial functions are the basis to obtain the lateral and orientation error of the vehicle with respect to the center of the lane.

2.6.1. Initial Conditions. As previously mentioned, the initial segmentation was derived basing on basic pattern $i$, whose width and edges are regarded as the initial values for the estimation process, i.e, $\hat{W}(t=$ $0)=W_{i}, \hat{y}_{l}(0)=y_{l i}, \hat{y}_{c}(0)=y_{c i}$, and $\hat{y}_{r}(0)=y_{r i}$, where $\hat{W}(t=0)$ represents the initial estimation of road width, and $W_{i}$ is the width of basic pattern $i$. On the other hand, $\hat{y}_{l}(0), \hat{y}_{c}(0), \hat{y}_{r}(0)$ are the initial estimations for the left edge, right edge, and skeleton lines of the road, respectively, while $y_{l i}, y_{r i}, y_{c i}$ stand for the left edge, right edge, and skeleton lines of basic pattern $i$.

\subsubsection{Estimation of the Skeleton Lines of the Road.}

The skeleton lines of the road at current time instant, $\hat{y}_{c}(t)$, is estimated based on the segmented low resolution image and the previously estimated road trajectory, $\hat{y}_{c}(t-1)$. Temporal correlation among measurements obtained at different instants of time is considered, as well as the number of data used to carry out the estimation, so as to enhance the road estimation process. Accordingly, a weighted-recursive least squares estimator with exponential decay is utilized for this purpose as initially proposed in Schneiderman et al. (1994). The analytical formulation of this filtering technique is quite similar to that of Kalman filter.

2.6.2.1. Data Measurement. The objective of this first stage is to extract a number of candidate points associated to the skeleton lines of the road at time instant $t$. For each line $k$ in the region of interest the maximum road width is determined based on the segmented image, as depicted in Fig. 15. The middle point of each maximal road width line $k$ is considered as candidate, and its coordinates $\left(y_{t, k}, x_{t, k}\right)$ are validated if the road width of line $k$ is greater than some threshold. This intends to account for noise rejection. 


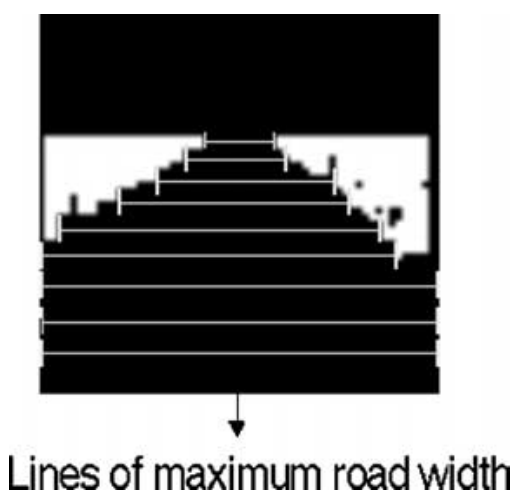

Figure 15. Maximum road width for each line in the region of interest.

2.6.2.2. Data Association and Validation. In order to provide the algorithm with noise rejection capacity, only candidate pixels whose distance to the previous estimation $\hat{y}_{c}(t-1)$ is under some threshold $V$ are validated and associated to the current measurement of the skeleton lines of the road. This permits to establish a validation area around the previous road model as depicted in Fig. 16. All measurements residing out of the validation area are discarded and regarded as invalid measurements.

Figure 17 depicts the points measured for a sequence of four images in a real scenario, using the proposed validation area, where a T-junction appears on the left hand side as a perturbation. The validation process should remove this perturbation and help obtain an appropriate road model update. For this purpose, measurements corresponding to lines where the maximum road width coincides with the image width

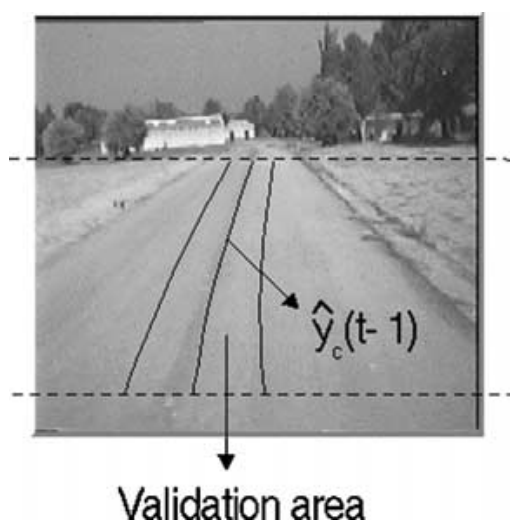

Figure 16. Validation area for measurements associated to the skeleton lines of the road.
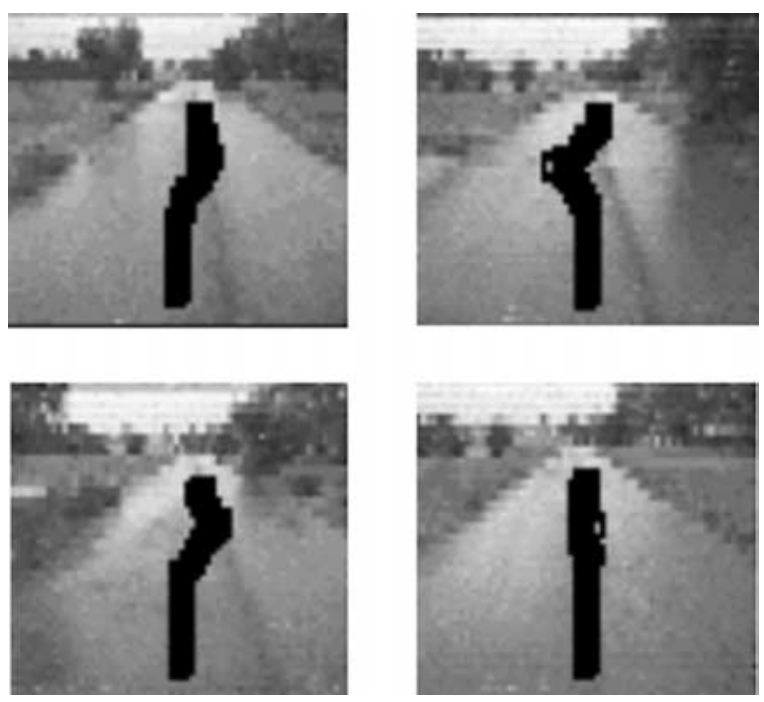

Figure 17. Data associated to the skeleton lines of the road in a sequence of images where a T-junction is traversed.

(i.e., lines where only road pixels are perceived), are also disregarded as valid measurements as long as those lines don't provide any information about the road edges. The effect of these false measurements can be graphically appreciated as straight segments in the bottom-middle part of the example images illustrated in Fig. 17.

2.6.2.3. Road Model Update. Measurements validated in the last stage constitute the starting point for updating the parabolic road model. As previously mentioned, a weighted-recursive least squares estimator with exponential decay is proposed to perform the estimation of the skeleton lines of the road. In spite of being a well-known and well-documented theory, we repeat the basic equations in this paper for completeness reasons. Thus, the estimation is realized in three steps as described below.

(a) Update prediction.

$$
\hat{z}(t)=\phi^{T}(t) \cdot \theta(t-1)
$$

(b) Update state covariance estimate.

$$
\begin{aligned}
& P(t)=\frac{1}{\lambda}[P(t-1)-[P(t-1) \phi(t) \\
& \left.\left.\quad \cdot\left(\lambda I+\phi^{T}(t) P(t-1) \phi(t)\right)^{-1} \cdot \phi^{T}(t) P^{T}(t-1)\right]\right]
\end{aligned}
$$


(c) Update state estimate.

$$
\theta(t)=\theta(t-1)+G(t) \cdot[z(t)-\hat{z}(t)]
$$

with

$$
\begin{aligned}
G(t) & =P(t-1) \phi(t) \cdot\left(\lambda I+\phi^{T}(t) P(t-1) \phi(t)\right)^{-1} \\
z(t) & =\left[\begin{array}{c}
y_{t, 1} \\
y_{t, 2} \\
\cdots \\
y_{t, N_{t}}
\end{array}\right] \quad \theta(t-1)=\left[\begin{array}{l}
c \\
b \\
a
\end{array}\right] \\
\phi^{T}(t) & =\left[\begin{array}{ccc}
1 & x_{t, 1} & x_{t, 1}^{2} \\
1 & x_{t, 2} & x_{t, 2}^{2} \\
\cdots & \cdots & \cdots \\
1 & x_{t, N_{t}} & x_{t, N_{t}}^{2}
\end{array}\right]
\end{aligned}
$$

where $\theta(t-1)$ represents the state estimation at the previous iteration of the algorithm, i.e., at time instant $t-\Delta T_{v}\left(\Delta T_{v}\right.$ is the sampling period of the road tracking algorithm), $\lambda$ is a scalar value that can vary in the range $0 \leq \lambda \leq 1$, and $P(t-1)$ stands for the state covariance at the previous time step. To achieve a proper tradeoff between robustness and transient response, $\lambda$ has been experimentally set to 0.7 , exhibiting an adequate performance in real tests. To illustrate the estimation process, Fig. 18 shows the results achieved upon a sequence of real images, using the proposed formulation.
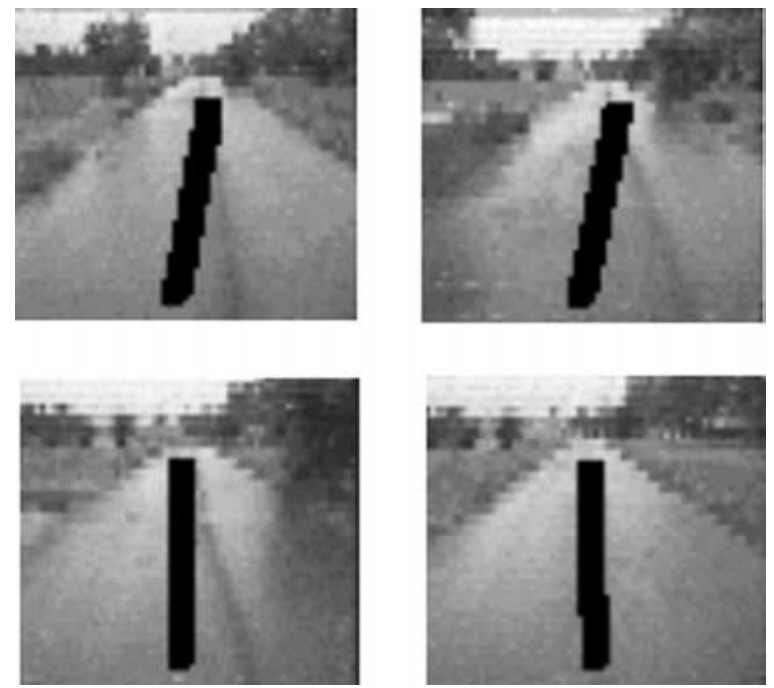

Figure 18. Estimation of the skeleton lines of the road in a sequence of real images.
2.6.3. Road Edges Estimation. The estimation of road edges is realized using the same filtering technique described in the previous section. Measurements for the left and right road edges are validated and enhanced based on three fundamental points: the estimation of the skeleton lines of the road at current time $t$, the estimation of road width at time $t-1$, and the slowly varying road width assumption. Thus, a validation area is also established for the left and right measurements, as depicted in Fig. 19. The locations of the left and right validation areas are based on the skeleton lines of the road estimated at time $t$, and the estimated width at time $t-1$. The left edge validation area is placed on the left, $\hat{W}(t-1) / 2$ meters away from the skeleton lines of the road $\hat{y}_{c}(t)$, while the right edge validation area is obviously located to the right, at $\hat{W}(t-1) / 2$ meters from $\hat{y}_{c}(t)$.

Consequently, for each line in the area of interest, the closest measurements to the middle of the left edge validation area, defined by $\hat{y}_{c}(t)-\hat{W}(t-1) / 2$, and right edge validation area, defined by $\hat{y}_{c}(t)+\hat{W}(t-1) / 2$, are considered and validated if the distance to the respective edge reference is below $V=1 \mathrm{~m}$. The estimations of the left and right edges are independently carried out basing on the validated measurements for each edge ( $N_{l t}$ points for the left edge and $N_{r t}$ for the right edge), while having different covariance matrices $\left(P_{l}(t)\right.$ for the left edge, $P_{r}(t)$ for the right edge). Upon the conclusion of the estimation process, vector $\theta_{l}(t)=\left(a_{l}, b_{l}, c_{l}\right)^{T}$ determines the coefficients of the parabolic polynomial that approximates the left road edge, while $\theta_{r}(t)=\left(a_{r}, b_{r}, c_{r}\right)^{T}$ determines the coefficients for the right edge. A complete example of image

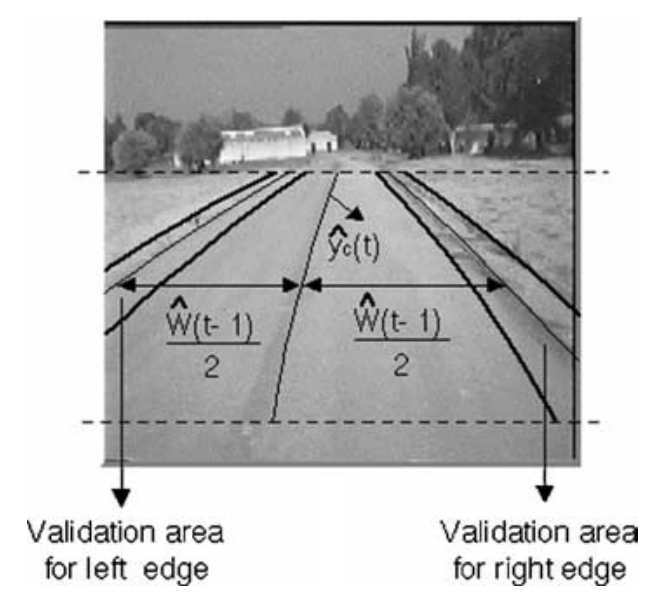

Figure 19. Validation areas for edge measurements. 


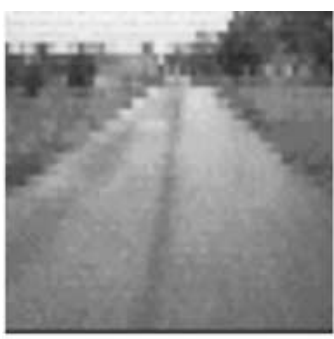

a)

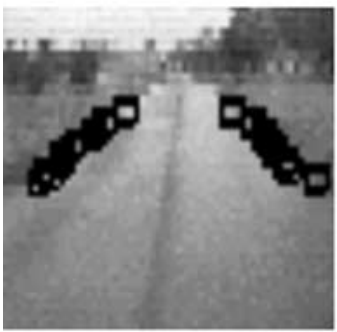

c)

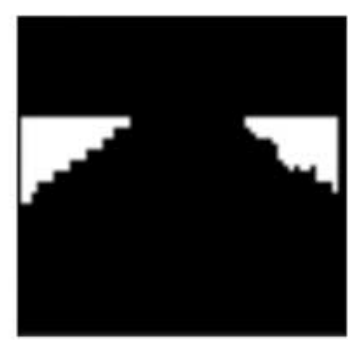

b)

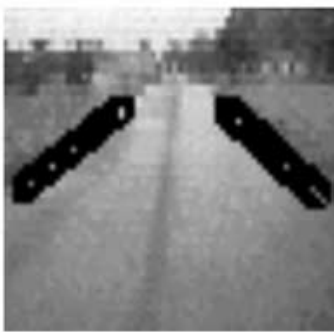

d)
Figure 20. A complete example. (a) Real image. (b) Segmentation of the region of interest. (c) Validated edge points. (d) Road edges estimation.

segmentation, edge points extraction, and road edges estimation is depicted in Fig. 20. Likewise, Fig. 21 depicts the estimation of road edges and skeleton lines in a sequence of images in which another vehicle is preceding the ego-vehicle, projecting its shadow on the pavement. The position of the lower part of the vehicle
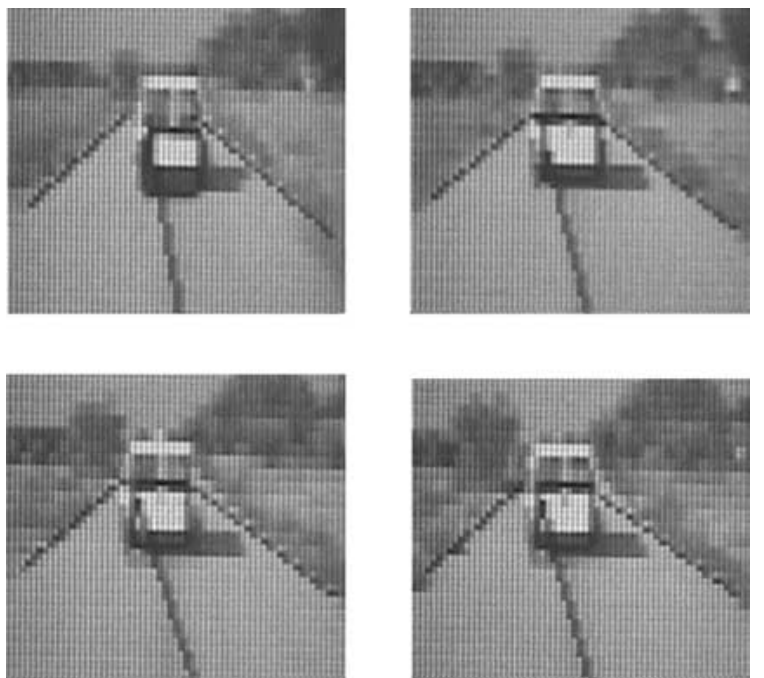

Figure 21. Estimation of road edges and skeleton lines in presence of another vehicle. is highlighted by a black square box in the image. The detailed description of the vehicle detection algorithm deployed in this work is out of the scope of this paper and can be found in Sotelo (2001). Although properly parameterized edge-based methods can also be used to detect the road edges (Lutzeler and Dickmanns, 2000), the consideration of color properties of the complete road surface, as proposed in this paper, serves also for vehicle detection reinforcement.

2.6.4. Road Width Estimation. The road width is an essential parameter in the complete road tracking scheme. Its estimation is realized on the basis of the previously mentioned slowly varying road width assumption. An individual road width measure $w_{i}$ is obtained for each line in the region of interest, by computing the difference between the left and right edges $\left(\hat{y}_{l}(t)_{\mid x=x_{i}}\right.$ and $\hat{y}_{r}(t)_{\mid x=x_{i}}$, respectively) as expressed in Eq. (20).

$$
w_{i}=\hat{y}_{r}(t)_{\mid x=x_{i}}-\hat{y}_{l}(t)_{\mid x=x_{i}}
$$

Measurements $w_{i}$ obtained in the image plane are properly corrected, using the calibration parameters, to yield real measurements in the $3 \mathrm{D}$ scene. The average road width measure at time $t, W(t)$, is computed using the individual measurements for each line, normalized by the number of valid measurements in the region of interest $N_{r d i}$, as in Eq. (21).

$$
W(t)=\frac{1}{N_{r d i}} \sum_{i=1}^{N_{r d i}} w_{r . i}
$$

The slowly varying road width assumption is incorporated using a recursive least squares based estimator, similar to those employed for the estimation of road edges. This permits to issue a smooth estimation of the road width. Its analytical formulation is presented below.

$$
\begin{aligned}
\hat{m}(t) & =\hat{W}(t-1) \\
P_{w}(t) & =\frac{1}{\lambda_{w}}\left(P_{w}(t-1)-K_{w}(t) P^{T}(t-1)\right) \\
\hat{W}(t) & =\hat{W}(t-1)+K_{w}(t)(W(t)-\hat{m}(t))
\end{aligned}
$$

with

$$
K_{w}(t)=P_{w}(t-1) /\left(\lambda_{w}+P_{w}(t-1)\right)
$$

where $\hat{m}(t)$ represents the prediction of the current state, and $P_{w}(t)$ stands for covariance of the estimated 


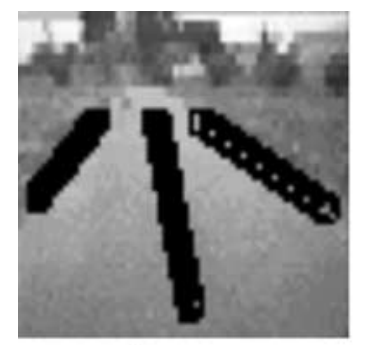

$\hat{W}=6.01 \mathrm{~m}$

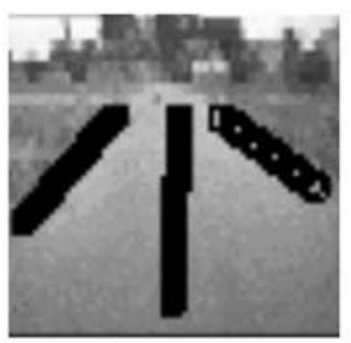

$\hat{W}$

$\hat{W}=5.98 \mathrm{~m}$

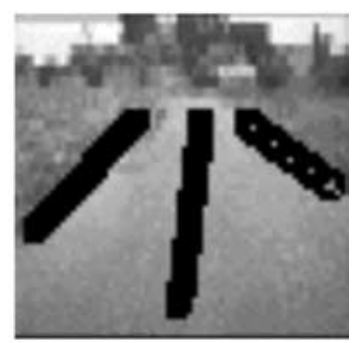

$\hat{W}=5.93 m$

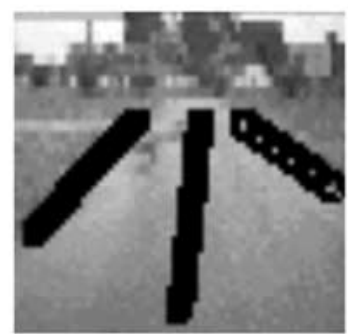

$\hat{W}=6.09 \mathrm{~m}$
Figure 22. Estimation of road edges and width in a sequence of images.

width. In practice, proper results have been achieved using $\lambda_{w}=0.8$. To graphically illustrate the whole process, Fig. 22 depicts a sequence of road images showing the estimation of the skeleton lines and edges of the road, as well as the estimated road width. Although the resolution of measurements is not high enough to obtain $1 \mathrm{~cm}$ accuracy in road width, it is possible to obtain such values using recursive estimators as described in this section.

\subsection{Road Color Features Update}

After completing the road edges and width estimation process, the HSI color features of the road pattern are consequently updated so as to account for changes in road appearance and illumination. Intuitively, pixels close to the skeleton lines of the road present color features that highly represent the road color pattern. Accordingly, a set of $N_{p}=8$ pixels in a region of $1 \mathrm{~m}$ surrounding the central estimation of the road, $\hat{y}_{c}(t)$, is randomly chosen as depicted in Fig. 23. Obviously, the selected pixels are only validated if they have been segmented as road pixels at the current iteration.

The HSI color features of the road pattern are properly averaged based on the individual HSI characteristics of the selected pixels, as shown in Eqs. (26)

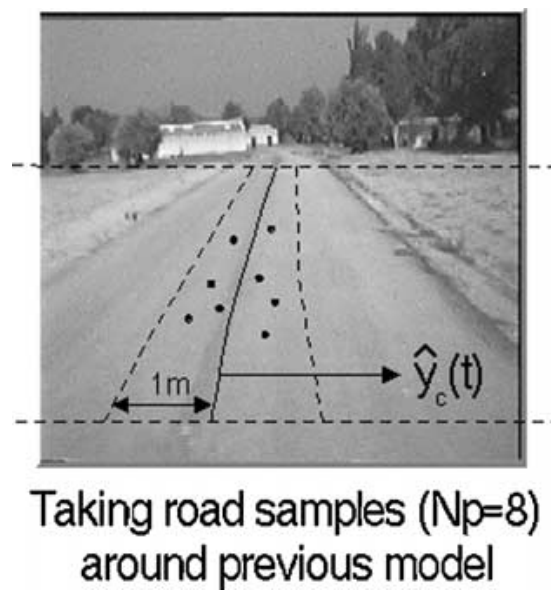

Figure 23. Random pixel selection for HSI road pattern update.

and (27).

$$
\begin{aligned}
C_{M} & =\sum_{k=1}^{N_{p}} S_{k} \cdot \cos \left(H_{k}\right) \\
S_{M} & =\sum_{k=1}^{N_{p}} S_{k} \cdot \operatorname{sen}\left(H_{k}\right) \\
H_{p} & =\arctan \left(S_{M} / C_{M}\right) \\
S_{p} & =\sqrt{C_{M}^{2}+S_{M}^{2}} \\
I_{p} & =\frac{1}{N_{p}} \sum_{k=1}^{N_{p}} I_{k}
\end{aligned}
$$

The adaptation process described in this section proves to be crucial in practice to keep the segmentation algorithm under stable performance upon illumination changing conditions and color varying asphalt. The complete road tracking scheme is graphically summarised in the flow diagram depicted in Fig. 24.

\subsection{Discussion of the Method}

The global objective of this section is to put the road tracking algorithm under test in varied real circumstances. Thus, we propose to analyse the system performance on different non-structured roads in strongly changing weather and illumination conditions as described below.

2.8.1. Non-Structured Roads. In a first set of trials, the road tracking algorithm is evaluated on a private test 


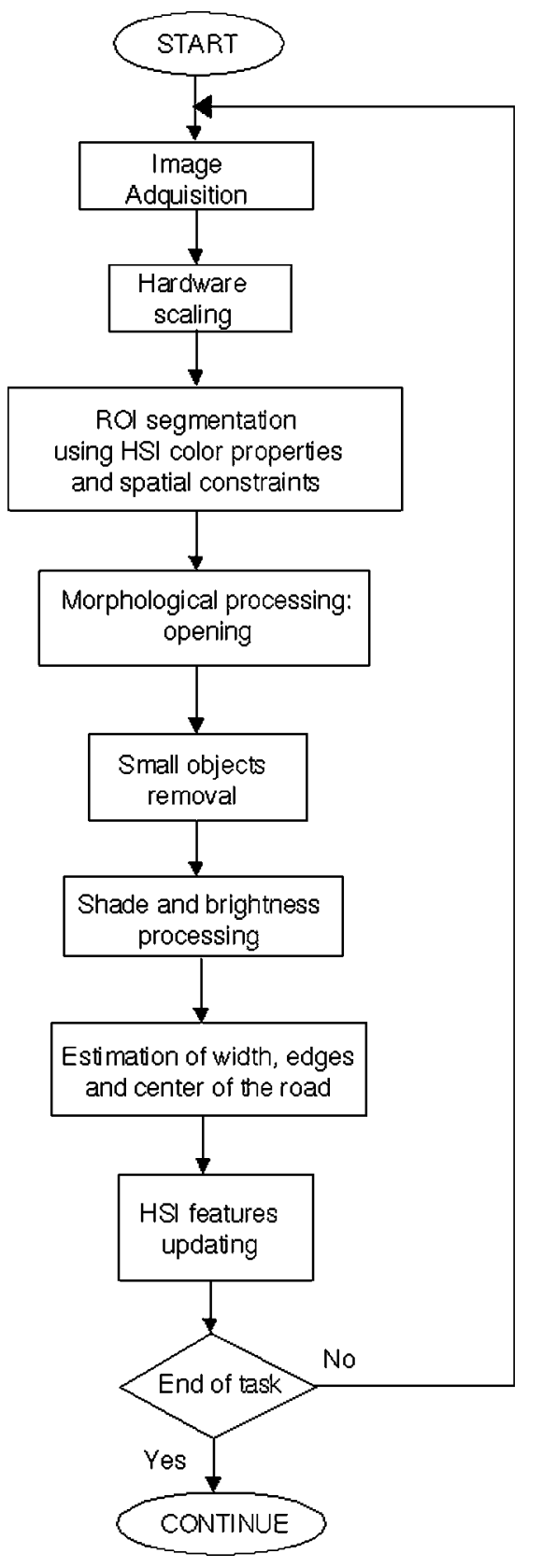

Figure 24. Road tracking flow diagram.

circuit. Figure 25 depicts an example of road segmentation obtained on this scenario. As appreciated from observation of Fig. 25, the road edges can be neatly distinguished in the segmented image, allowing a clear estimation in real experiments.

In a second trial, similar experiments were conducted on rural roads. As observed in Fig. 26, a central lane
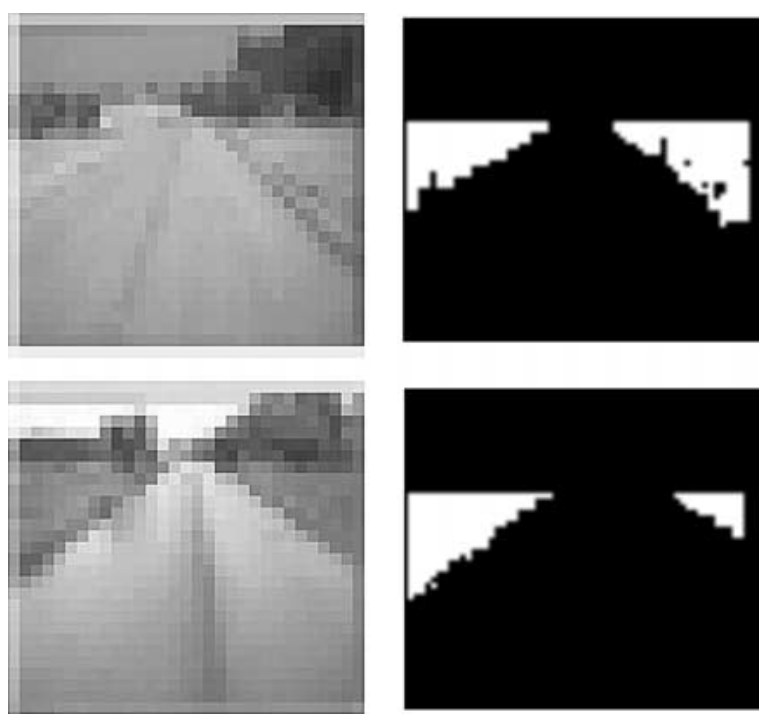

Figure 25. Road segmentation obtained on a private circuit.
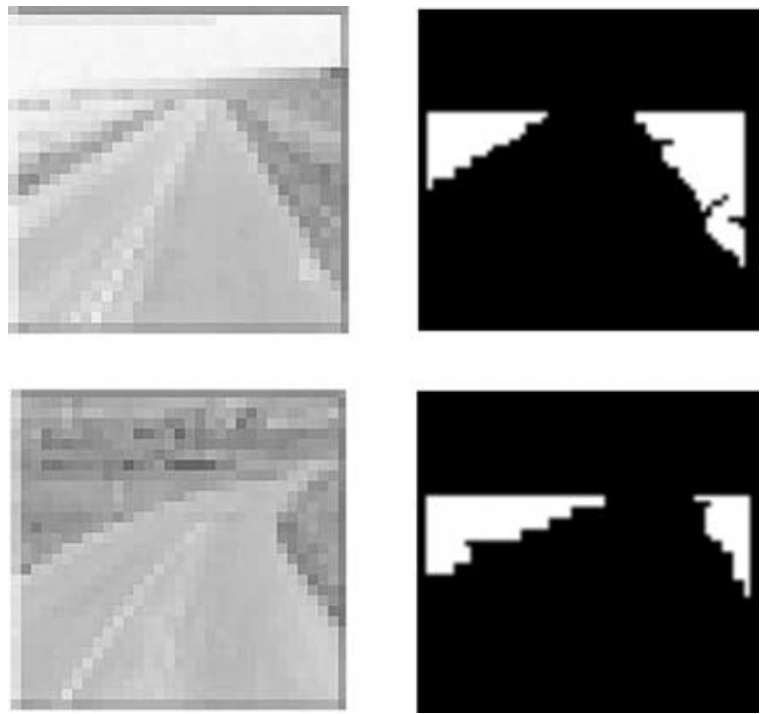

Figure 26. Segmentations obtained on rural roads.

marker is clearly painted on the asphalt, while no lane markers are present at all near the road edges. In spite of these structural conditions, the segmentations depicted in Fig. 26 strongly support the ability of the road tracking algorithm to successfully perform in this kind of scenario. On the other hand, as can be appreciated in Fig. 26, small focal length cameras should be used in order to allow for high speed driving in wide roads with high curvature. Otherwise, the driving behavior would lead to oscillations. 

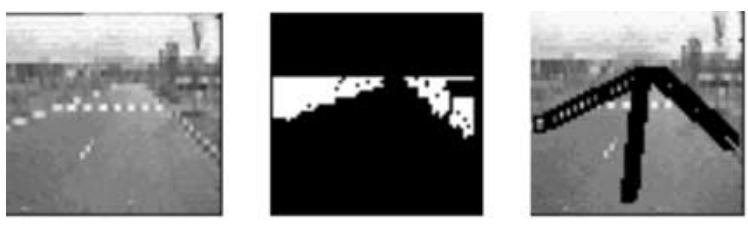

a)
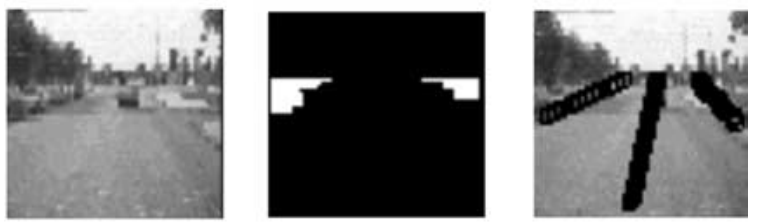

b)

Figure 27. Segmentation and road edges estimation on urban areas in presence of (a) a zebra crossing and (b) other vehicles.

The system has also been evaluated on a University Campus using a set of recorded images. Thus, Fig. 27 depicts two different and representative situations on urban driving. On one hand, Fig. 27(a) shows the segmentation and road edges estimation in presence of a zebra crossing. As can be observed, the segmentation algorithm provides an efficient filtering for this kind of perturbation, while preserving correct estimation of the road edges. During the color model update, only those pixels that were segmented as road before the morphological filtering step can be chosen to enter the model. Thus, white lines painted on the road as well as zebra crossing lines don't affect the model update process. On the other hand, they don't affect either the road segmentation process itself, as white lines and zebra crossings lines are segmented as road after morphological filtering and shadows and brightness attenuation due to its high intensity value. The results obtained in presence of other vehicles parked on the left hand side of the road are illustrated in Fig. 27(b). Correct segmentation and edges estimation are also achieved in this case.

In a final trial, the road tracking scheme is put under evaluation on roads without asphalt. Accordingly, the edges of a narrow rural path are estimated basing on the road segmentation, as depicted in Fig. 28.

\subsubsection{Robustness Against Environmental and} Weather Conditions. In this section, the performance of the road tracking algorithm is evaluated under different environmental and weather conditions, so as to
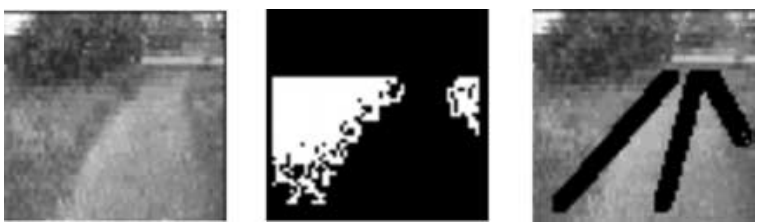

Figure 28. Segmentation and road edges estimation on a rural path without asphalt.

verify the validity and generality of the segmentation and updating scheme. All tests described in this section were conducted on a private circuit.

2.8.2.1. Sunny Conditions. The excessive amount of luminance becomes a major problem when dealing with road images in a sunny day. All pixels in the image tend to have similar intensity values, and thus, color differences in the HSI chromatic plane become crucial for segmentation purposes. In spite of having achieved correct performance in real experiments under sunny conditions in general terms, a few remarks must be pointed out.

There exist limitations in the schedule of applicability due to direct incidence of sunrays onto the camera lens when driving into the sun, just after sunrise and before sunset on strongly sunny days. Accordingly, autonomous navigation becomes dangerous and non-advisable under these conditions that obviously depend on the latitude and the season of the year.

The presence of strong and large shadows on the road especially after sunrise may complicate the segmentation process to the extent of making recommendable to decrease vehicle velocity, in order to avoid driving oscillations. Nevertheless, transitions from shaded to sunny areas as well as navigation on completely shaded zones are adequately managed by the segmentation and updating algorithm out of the critical hours before mentioned.

2.8.2.2. Cloudy and Rainy Conditions. The amount of intensity in the image strongly decreases on cloudy and rainy days. Paradoxically, this circumstance eases the discrimination process between road and non-road pixels, as color differences become larger. Figure 28 depicts a typical example of image segmentation and road edges estimation on cloudy conditions. As can be observed, the high quality of the road segmentation shown in Fig. 29 supports the previous discussion. 

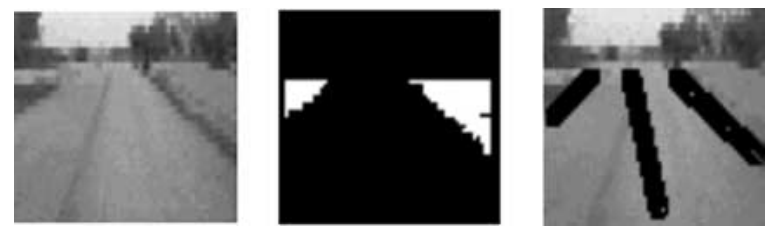

Figure 29. Segmentation and edges estimation on cloudy conditions.
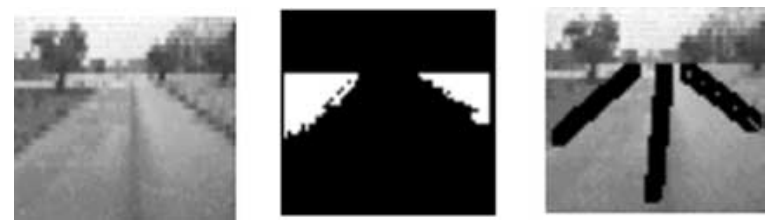

Figure 30. Segmentation and edges estimation on rainy conditions.
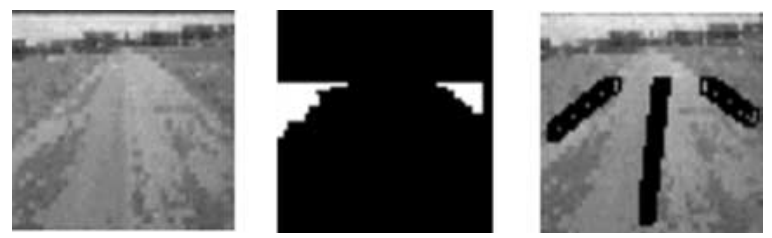

Figure 31. Segmentation and road edges estimation on post-rainy conditions.

Likewise, the road takes quite a characteristic color on rainy days because of the water on it. This also contributes to a better and easier separation between road and non-road pixels, as graphically demonstrated in Fig. 30, where both the image segmentation and road edges estimation are illustrated for a typical rainy scene.

However, the most dangerous situation takes place after the rain stops, as the asphalt gets dry in a nonhomogeneous manner. This situation derives in the appearance of dark spots on the road due to wet areas, as depicted in Fig. 31. Fortunately, the segmentation process (including the small blobs removal stage) properly manages these circumstances allowing to obtain high quality segmentations, as shown in Fig. 31.

2.8.2.3. Foggy Days. In general, autonomous navigation is not advisable on foggy days, even for humans. In spite of this, proper segmentations can be obtained under non-heavy foggy conditions, as depicted in Fig. 32. In this case the camera orientation and region of interest were slightly modified so as to
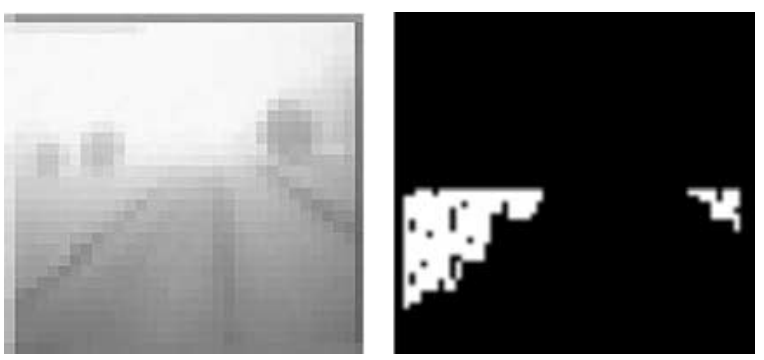

Figure 32. Road segmentation on non-heavy foggy conditions.

cover the bottom part of the image, where the contrast is high enough for color segmentation under these circumstances.

\section{Implementation and Results}

The complete navigation system described in the previous sections has been implemented on the so-called Babieca prototype vehicle, depicted in Fig. 1, that has been modified to allow for automatic velocity and steering control at a maximum speed of $90 \mathrm{~km} / \mathrm{h}$, using the non linear control law developed in Sotelo (2001). Babieca is equipped with a color camera, a Pentium PC, and a set of electronic devices to provide actuation over the accelerator and steering wheel, as well as to encode the vehicle velocity and steering angle. The color camera provides standard PAL video signal at $25 \mathrm{~Hz}$ that is processed by a Meteor frame grabber installed on a 120 $\mathrm{MHz}$ Pentium running the Real Time Linux operating system. After implementing the complete navigation system under Real Time Linux using a pre-emptive scheduler (Barabanov, 1997), the lane tracking vision based task gets executed at 10 frames/s. Practical experiments were conducted on a private circuit located at the Instituto de Automática Industrial in Arganda del Rey (Madrid). The circuit is composed of several streets and intersections, trying to emulate an urban quarter. Babieca has ran over hundreds of kilometers in lots of successful autonomous missions carried out along the test circuit. As stated in Section 1, a live demonstration exhibiting the system capabilities on autonomous driving was also carried out during the IEEE Conference on Intelligent Vehicles 2002, in a private circuit located at Satory (Versailles), France. In order to complete the graphical results depicted in the previous sections, and to illustrate the global behavior of the complete navigation system implemented on Babieca, some general results are shown next. Thus, Fig. 33 


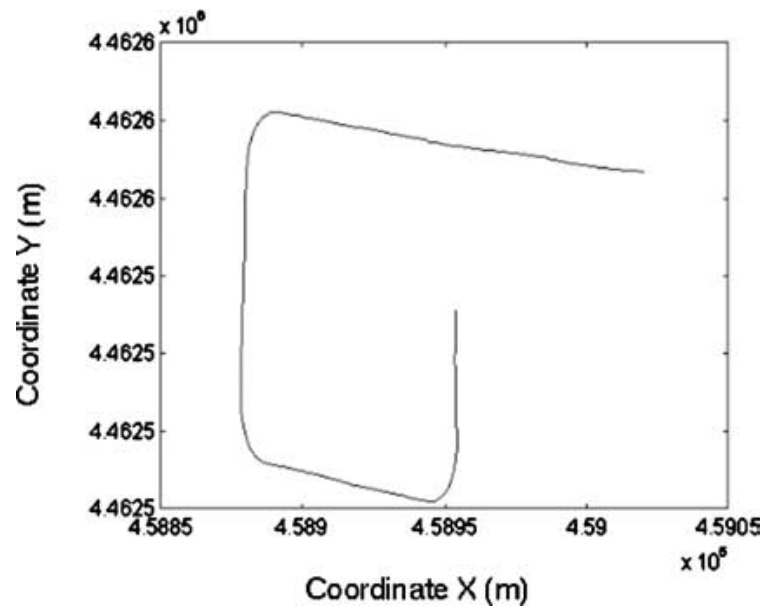

Figure 33. Real trajectory followed by the vehicle in an autonomous mission. shows the $2 \mathrm{D}$ real trajectory followed by Babieca (using UTM coordinates as measured by a DGPS receiver onboard the vehicle), including several sharp bends.

On the other hand, various practical trials were conducted so as to test the validity of the control law for different initial conditions in real circumstances. During the tests, the reference vehicle velocity is assumed to be kept constant by the velocity controller. Figures 34 and 35 show the transient response of the vehicle lateral and orientation error for reference velocities of $20 \mathrm{~km} / \mathrm{h}$ and $50 \mathrm{~km} / \mathrm{h}$, respectively. Relative measurements during the tests were obtained from a DGPS receiver in order to support the ability of the vision-based algorithm to correctly locate the vehicle in the lane. In all cases, the vehicle starts the run at an initial lateral error of about $1 \mathrm{~m}$ and an initial orientation error in the
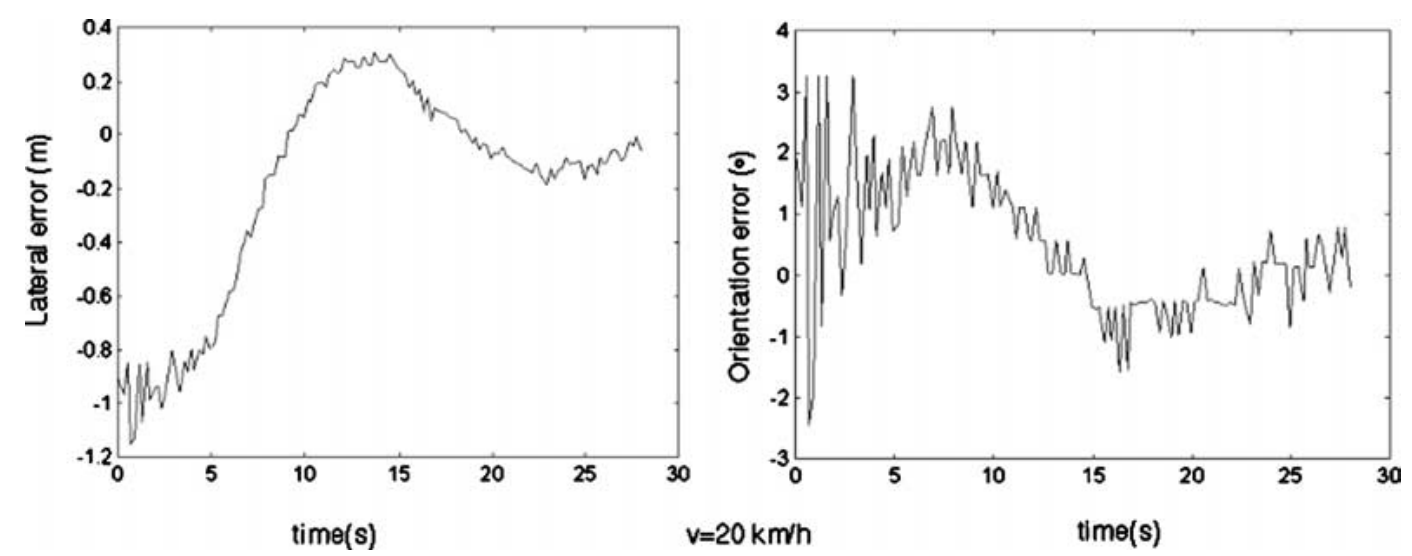

Figure 34. Transient response of the lateral and orientation error for $v=20 \mathrm{~km} / \mathrm{h}$.
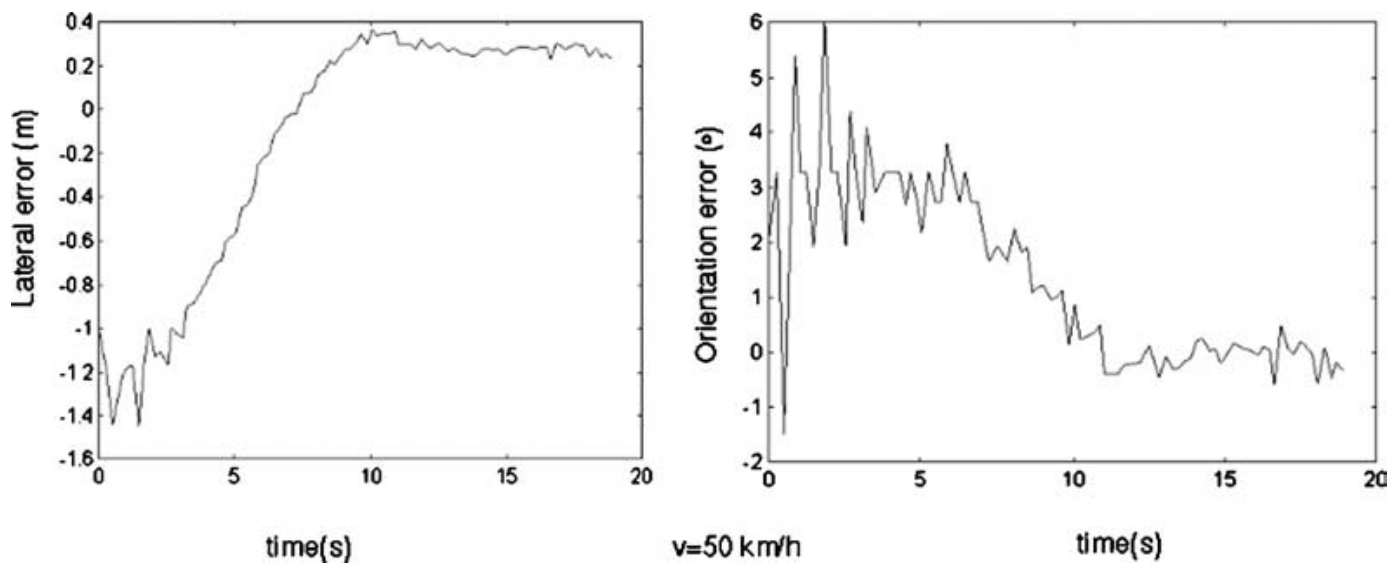

Figure 35. Transient response of the lateral and orientation error for $v=50 \mathrm{~km} / \mathrm{h}$. 
range $\pm 5^{\circ}$. As can be clearly appreciated, the steady state response of the system is satisfactory for both experiments. Thus, the lateral error is bound to $\pm 5 \mathrm{~cm}$ at low speeds and $\pm 25 \mathrm{~cm}$ at $\mathrm{v}=50 \mathrm{~km} / \mathrm{h}$, while the absolute orientation error in steady state remains below $1^{\circ}$ in all cases.

To complete these results a wide set of video files demonstrating the operational performance of the system in real tests can be retrieved from ftp://www. depeca.uah.es/pub/vision.

\section{Conclusions}

The road segmentation algorithm based on the HSI color space and 2D-spatial constraints, as described in this work, has successfully proved to provide correct estimations for the edges and width of non-structured roads, i.e., roads without lane markers. The practical results discussed above also support the validity of the method for different environmental and weather conditions, as demonstrated so far. On the contrary, there exist some limitations in the use of the road tracking algorithm just after sunrise and before sunset in very sunny days, but only when driving into the sun due to direct incidence of sunrays onto the camera plane. Otherwise, there will be long shadows but the system performs well. The most remarkable feature of the road tracking scheme described in this work is its ability to correctly deal with non-structured roads by performing a non-supervised color-based road segmentation process. The system may be used for driver assistance or for the implementation of autonomous guidance. For this purpose, the position of the egovehicle in the lane (lateral and orientation error) is computed using as references the estimated skeleton lines of the road and its edges. The validity and generality of the algorithm presented in this paper is plausibly supported by real experiments. In fact, as previously stated, the complete system has been successfully tested on two different private circuits under different weather conditions. Nonetheless, a lot of work still remains to be done until a completely robust and reliable autonomous system can be fully deployed in real conditions.

\section{Acknowledgments}

This work has been funded by the Departamento de Electrónica of the UAH, and the generous support of the Instituto de Automática Industrial of the CSIC (Consejo Superior de Investigaciones Cientificas).

\section{References}

Barabanov, M. 1997. A Linux-based real-time operating system. Master of Science Thesis in Computer Science. New Mexico Institute of Mining and Technology. New Mexico.

Bertozzi, M. and Broggi, A. 1998. GOLD: A parallel real-time stereo vision system for generic obstacle and lane detection. IEEE Transactions on Image Processing, 7(1):62-81.

Bertozzi, M., Broggi, A., and Fascioli, A. 2000. Vision-based intelligent vehicles: State of the art and perspectives. Robotics and Automation Systems, 32:1-16.

Broggi, A., Bertozzi, M., Fascioli, A., and Conte, G. 1999. Automatic Vehicle Guidance: The Experience of the ARGO Autonomous Vehicle. World Scientific.

Chapuis, R., Aufrre, R., and Chausse, F. 1999. Recovering the 3D shape of a road by vision. In Proceedings of the International Conference on Image Processing Applications, Manchester, UK, pp. 686-690.

Crisman, J.D. and Thorpe, C.E. 1991. UNSCARF, a color vision system for the detection of unstructured roads. In Proceedings of the IEEE International Conference on Robotics and Automation, Sacramento.

De Pedro, T., Garcia, R., Gonzalez, C., Naranjo, J.E., Reviejo, J., and Sotelo, M.A. 2001. Vehicle automatic driving system based on GNSS. In Proceedings of the International Conference on Intelligent Vehicles, Seville, Spain.

Dickmanns, E.D. and Zapp, A. 1986. A curvature-based scheme for improving road vehicle guidance by computer vision. In Mobile Robots, SPIE Proc., Vol. 727, Cambridge, Mass: Paris, pp. 161168.

Dickmanns, E.D. and Mysliwetz, B. 1992. Recursive 3-D road and relative ego-state recognition. In IEEE Transactions PAMI, Vol. 14, No. 2, Special Issue on 'Interpretation of 3-D Scenes', pp. 199-213.

Dickmanns, E.D., Behringer, R., Dickmanns, D., Hildebrant, T., Mauer, M., Thomanek, F., and Shielhlen, J. 1994. The seeing passenger car 'VaMoRs-P'. In Proc. of Int. Symp. on Intelligent Vehicles, Paris.

Franke, U., Gavrila, D., Gorzig, S., Lindner, F., Paitzold, F., and Wohler, C. 1998. Autonomous driving goes downtown. In Proceedings of the IEEE Intelligent Vehicles Symposium, Stuttgart, Germany.

Gonzales, R.C. and Wood, R.E. 1992. Digital Image Processing. Addison-Wesley: Reading, MA.

Gregor, R., Lutzeler, M., and Dickmanns, E.D. 2001. EMSVision: Combining on- and off-road driving. In Proc. SPIE Conf. on Unmanned Ground Vehicle Technology III, Aerosense 01.

Gregor, R., Lutzeler, M., Pellkofer, M., Siedersberger, K.H., and Dickmanns, E.D. 2001. A vision system for autonomous ground vehicles with a wide range of maneuvering capabilities. In Proc. ICVS, Vancouver.

Gregor, R., Lutzeler, M., Pellkofer, M., Siedersberger, K.H., and Dickmanns, E.D. 2002. EMS-Vision: A perceptual system for autonomous vehicles. IEEE Transactions on Intelligent Transportation Systems, 3(1):48-59. 
Hebert, M.H., Thorpe, C., and Stentz, A. 1997. Intelligent Unmanned Ground Vehicles. Autonomous Navigation Research at Carnegie Mellon.

Ikonomakis, N., Plataniotis, K.N., and Venetsanopoulos. 2000. Color image segmentation for multimedia applications. Journal of Intelligent and Robotic Systems 28:5-20.

Kim, K.J., Oh, S.Y., Kim, S.W., Jeong, H., Lee, C.N., Kim, B.S. and Kim, C.S. 1995. An autonomous land vehicle PRV II: Progresses and performance enhancement. In Proceedings of the IEEE Intelligent Vehicles Symposium, Detroit.

Kluge, K. and Thorpe, C. 1993. Intersection detection in the YARF road following system. Ingelligent Autonomous Systems, 3. Pittsburgh, PA, USA.

Lutzeler, M. and Dickmanns, E.D. 1998. Road recognition with MarVEye. In Proceedings of the IEEE Intelligent Vehicles Symposium, Stuttgart, Germany.

Lutzeler, M. and Dickmanns, E.D. 2000. EMS-Vision: Recognition of intersections on unmarked road networks. In International Symposium on Intelligent Vehicles (IV'2000), Dearborn, MI.

Pomerleau, D.A. 1993. Neural Network Perception for Mobile Robot Guidance. Kluwer Academic Publishers: Boston.

Pomerleau, D.A. and Jockem, T.M. 1996. Rapidly adapting machine vision for automated vehicle steering. IEEE Expert 11(2).

Rodriguez, F.J., Mazo, M., and Sotelo, M.A. 1998. Automation of an industrial fork lift truck, guided by artificial vision in open environments. Autonomous Robots, 5:215-231.

Schneiderman, H. and Nashman, M. 1994. A discriminating feature tracker for vision-based autonomous driving. IEEE Transactions on Robotics and Automation, 10(6).

Schneiderman, H., Wavering, A.J., Nashman, M., and Lumia, R. 1994. Real time model-based visual tracking. In International Workshop on Intelligent Robotic Systems.

Sotelo, M.A., Alcalde, S., Reviejo, J., Naranjo, J.E., Garcia, R., de Pedro, T., and Gonzalez, C. 2001. Vehicle fuzzy driving based on DGPS and vision. In 9th IFSA World Congress and 20th NAFIPS International Conference, Vancouver, Canada.

Sotelo, M.A. 2001. Sistema de Navegación Global Aplicado al Guiado de un Vehiculo Autónomo Terrestre en Entornos Exteriores Parcialmente Conocidos. PhD Dissertation, University of Alcala.

Thorpe, C. 1990. Vision and Navigation: The Carnegie Mellon Navlab. Kluwer Academic Publishers: Boston.

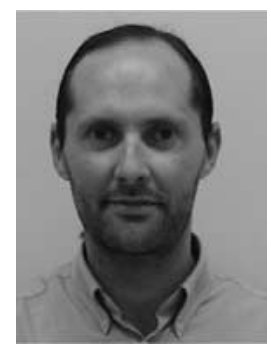

Miguel Ángel Sotelo received the Dr. Ing. degree in Telecommunication Engineering in 1996 from the Technical University of Madrid, and the Ph.D. degree in Telecommunication Engineering in 2001 from the University of Alcalá, Alcalá de Henares, Madrid, Spain. From 1993 to 1994 he has been a Researcher at the Department of
Electronics, University of Alcalá, where he is currently an Associate Professor. His research interests include real-time computer vision and control systems for autonomous and assisted intelligent vehicles, as well as vision and DGPS based global navigation systems. He has been recipient of the Best Research Award in the domain of Automotive and Vehicle Applications in Spain, in 2002. He is the author of more than 40 publications in international journals, book chapters, and conference proceedings.

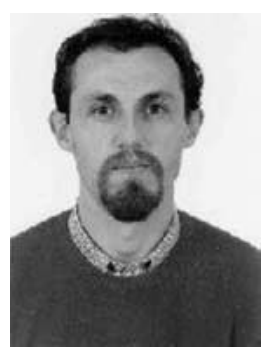

Francisco Javier Rodríguez received a Ph.D. in Electronics Engineering from the University of Alcalá in 1997, a Telecommunication Engineering degree from the Technical University of Madrid, in 1990, and a Technical Telecommunication Engineering degree in 1985. He has worked in the private electronic industry for two years; since 1986 he has been a Lecturer in the Electronics Department of the University of Alcalá, where he is an Associate Professor. His current work covers the areas of monitoring and control, real time processing, autonomous vehicles, and embedded systems.

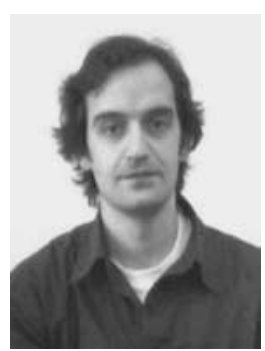

Luis Magdalena received the M.S. and Ph.D degrees in Telecommunication Engineering, both from the Technical University of Madrid, Spain, in 1988 and 1994, respectively. He is an Associate Professor in Computer Science at the Department of Applied Mathematics of the Technical University of Madrid. His research interests include Soft Computing and its application, particularly in the field of robotics. He is co-author of the book "Genetic Fuzzy Systems: Evolutionary Tuning and Learning of Fuzzy Knowledge Bases" (World Scientific, 2001). He is co-editor of the books "Technologies for Constructing Intelligent Systems" (two volumes, Phisica Verlag, 2002) and "Fuzzy Modelling and the Interpretability-Accuracy Trade-off" (two volumes, Phisica Verlag, 2002). He has co-edited special issues of the journals "International Journal of Intelligent Systems", "Information Sciences" and "Fuzzy Sets and Systems". Since 2001 he is the President of the "European Society for Fuzzy Logic and Technology". He served as co-chair of the "Eight International Conference on Information Processing and Management of Uncertainty in Knowledge-Based Systems" (Madrid, 2000). 


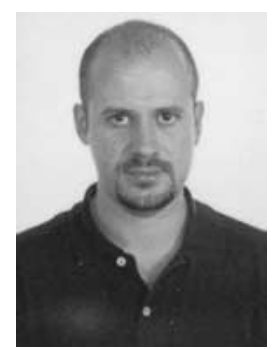

Luis Miguel Bergasa received a Ph.D. degree in Telecommunication Engineering from the University of Alcalá in 1999, a degree in Telecommunication Engineering from the Technical University of Madrid in 1995, and a degree in Technical Telecommunication Engineering from the University of Alcalá in 1989. He is currently an Associate Professor in the Department of Electronics at the University of Alcalá (Spain). His research interests include computer vision, robotics, and intelligent control.

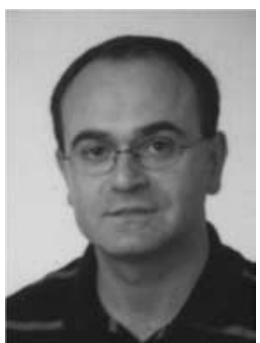

Luciano Boquete received a Ph.D. degree in Telecommunication Engineering in 1998, a degree in Telecommunication Engineering in 1994 and a degree in Technical Telecommunication Engineering in 1987. He is currently an Associate Professor in the Electronics Department at the University of Alcalá (Spain). His research interests include bioengineering, computer vision, system control, and neural networks. He is the author of more than 50 referred publications in international jounals, book chapters, and conference proceedings. 